\title{
A New Series of Three-Dimensional Chaotic Systems with Cross-Product Nonlinearities and Their Switching
}

\author{
Xinquan Zhao, ${ }^{1}$ Feng Jiang, ${ }^{1}$ Zhigang Zhang, ${ }^{2}$ and Junhao $\mathrm{Hu}^{3}$ \\ ${ }^{1}$ School of Statistics and Mathematics, Zhongnan University of Economics and Law, Wuhan 430073, China \\ ${ }^{2}$ Department of Statistics and Applied Mathematics, Hubei University of Economics, Wuhan 430205, China \\ ${ }^{3}$ School of Mathematics and Statistics, South-Central University for Nationalities, Wuhan 430074, China
}

Correspondence should be addressed to Xinquan Zhao; zhaoxq56@gmail.com

Received 31 October 2012; Accepted 25 December 2012

Academic Editor: Xinzhi Liu

Copyright (C) 2013 Xinquan Zhao et al. This is an open access article distributed under the Creative Commons Attribution License, which permits unrestricted use, distribution, and reproduction in any medium, provided the original work is properly cited.

\begin{abstract}
This paper introduces a new series of three-dimensional chaotic systems with cross-product nonlinearities. Based on some conditions, we analyze the globally exponentially or globally conditional exponentially attractive set and positive invariant set of these chaotic systems. Moreover, we give some known examples to show our results, and the exponential estimation is explicitly derived. Finally, we construct some three-dimensional chaotic systems with cross-product nonlinearities and study the switching system between them.
\end{abstract}

\section{Introduction}

Since Lorenz discovered the well-known Lorenz chaotic system, many other chaotic systems have been found, including the well-known Rössler system and Chua's circuit, which serve as models of the study of chaos [1-12].

The Lorenz system plays an important role in the study of nonlinear science and chaotic dynamics [13-18]. We know that it is extremely difficult to obtain the information of chaotic attractor directly from system. Most of the results in the literature are based on computer simulations. When calculating the Lyapunov exponents of the system, one needs to assume that the system is bounded in order to conclude chaos. Therefore, the study of the globally attractive set of the Lorenz system is not only theoretically significant but also practically important. Moreover, Liao et al. $[19,20]$ gave globally exponentially attractive set and positive invariant set for the classical Lorenz system and the generalized system by constructive proofs. In addition, Yu et al. [21] studied the problem of invariant set of systems, which was considered as a more generalized Lorenz system.

In this paper, we consider the following three-dimensional autonomous systems with cross-product nonlinearities:

$$
\dot{x}=A x+f(x)+C,
$$

where $x^{T}=\left(x_{1}, x_{2}, x_{3}\right)$ and

$$
\begin{gathered}
A=\left[\begin{array}{lll}
a_{11} & a_{12} & a_{13} \\
a_{21} & a_{22} & a_{23} \\
a_{31} & a_{32} & a_{33}
\end{array}\right] ; \quad C=\left[\begin{array}{l}
c_{1} \\
c_{2} \\
c_{3}
\end{array}\right] ; \quad f(x)=\left[\begin{array}{l}
x^{T} B_{1} x \\
x^{T} B_{2} x \\
x^{T} B_{3} x
\end{array}\right] ; \\
B_{i}=\left[\begin{array}{lll}
b_{i 11} & b_{i 12} & b_{i 13} \\
b_{i 21} & b_{i 22} & b_{i 23} \\
b_{i 31} & b_{i 32} & b_{i 33}
\end{array}\right], \quad i=1,2,3
\end{gathered}
$$

with $a_{i j}, b_{i j k}, c_{i} \in R, i, j, k=1,2,3$. This second-order dynamic system may be regarded as the most general Lorenz system. For such system, we can choose Lyapunov function:

$$
\begin{gathered}
V(X(t))=\frac{1}{2}\left[\left(\lambda_{1} x_{1}-d_{1}\right)^{2}+\left(\lambda_{2} x_{2}-d_{2}\right)^{2}\right. \\
\left.+\left(\lambda_{3} x_{3}-d_{3}\right)^{2}\right],
\end{gathered}
$$

which is obviously positive definite and radially unbounded, where $d_{i}, \lambda_{i}, i=1,2,3$ are undetermined parameters. In this paper, we will study this more general Lorenz system (1) than the classical system and the generalized Lorenz system. The result obtained contains earlier results as its special cases.

This paper is organized as follows. In Section 2, we define the globally exponentially attractive set and positive invariant 
set and the globally conditional exponentially attractive set and positive invariant set of the three-dimensional chaotic systems with cross-product nonlinearities. In Section 3, the qualitative analysis of the exponentially attractive set and positive invariant set of the chaotic systems has been done. In Section 4, we also suggest an idea to construct chaotic systems, and some new chaotic systems and switched chaotic systems are illustrated.

\section{Preliminaries}

In this section, we present some basic definitions which are needed for proving all theorems in the next section. For convenience, denote $X:=\left(x_{1}, x_{2}, x_{3}\right)$ and $X(t):=$ $X\left(t, t_{0}, X_{0}\right)$.

Definition 1. For the three-dimensional autonomous systems with cross-product nonlinearities (1), if there exists compact (bounded and closed) set $\Omega \in R^{3}$ such that for all $X_{0} \in R^{3}$, the following condition: $\rho(X(t), \Omega):=\inf _{Y \in \Omega}\|X(t)-Y\| \rightarrow 0$ as $t \rightarrow+\infty$, holds, then the set $\Omega$ is said to be globally attractive. That is, system (1) is ultimately bounded; namely, system (1) is globally stable in the sense of Lagrange or dissipative with ultimate bound.

Furthermore, if for all $X_{0} \in \Omega_{0} \subseteq \Omega \subset R^{3}, X\left(t, t_{0}, X_{0}\right) \subseteq$ $\Omega_{0}$, then $\Omega_{0}$ for $t \geq 0$ is called the positive invariant set of the system (1).

Definition 2. For the three-dimensional autonomous systems with cross-product nonlinearities (1), if there exist compact set $\Omega \subset R^{3}$ such that for all $X_{0} \in R^{3}$ and constants $M>$ $0, \alpha>0$ such that $\rho(X(t), \Omega) \leq M e^{-\alpha\left(t-t_{0}\right)}$, then the threedimensional autonomous systems with cross-product nonlinearities system (1) are said to have globally exponentially attractive set, or the system (1) is globally exponentially stable in the sense of Lagrange, and $\Omega$ is called the globally exponentially attractive set.

Definition 3. For the three-dimensional autonomous systems with cross-product nonlinearities (1), if there exist compact set $\Omega \subset R^{3}$, a constant $\alpha>0$, and a bounded function $M\left(x_{0}, t\right)>0$ on $t$, as $t \geq t_{0}$, such that $\rho(X(t), \Omega) \leq$ $M\left(x_{0}\right) e^{-\alpha\left(t-t_{0}\right)}$, where $M\left(x_{0}\right)=\sup M\left(x_{0}, t\right), t \geq t_{0}$, then the system (1) is said to have globally conditional exponentially attractive set, or the system (1) is globally conditional exponentially stable in the sense of Lagrange, and $\Omega$ is called the globally conditional exponentially attractive set.

In general, from the definition we see that a globally exponential attractive set is not necessarily a positive invariant set. But our results obtained in the next section indeed show that a globally exponentially attractive set is a positive invariant set.

Note that it is difficult to verify the existence of $\Omega$ in Definition 2. Since the Lyapunov direct method is still a powerful tool in the study of asymptotic behaviour of nonlinear dynamical systems, the following definition is more useful in applications.
Definition 4. For three-dimensional autonomous systems with cross-product nonlinearities (1), if there exist a positive definite and radially unbounded Lyapunov function $V(X(t))$ and positive numbers $L>0, \alpha>0$ such that the following inequality

$$
V(X(t))-L \leq\left(V\left(X_{0}\right)-L\right) e^{-\alpha\left(t-t_{0}\right)}
$$

is valid for $V(X(t))>L\left(t \geq t_{0}\right)$, then the system (1) is said to be globally exponentially attractive or globally exponentially stable in the sense of Lagrange, and $\Omega:=\{X \mid V(X(t)) \leq$ $\left.L, t \geq t_{0}\right\}$ is called the globally exponentially attractive set.

Definition 5. For the three-dimensional autonomous systems with cross-product nonlinearities (1), if there exist a positive definite and radially unbounded Lyapunov function $V(X(t))$ and a bounded function $L\left(x_{0}, t\right)>0$, on $t$, as $t \geq t_{0}$, and $\alpha>0$ such that the following inequality

$$
V(X(t))-L\left(x_{0}\right) \leq\left(V\left(X_{0}\right)-L\left(x_{0}\right)\right) e^{-\alpha\left(t-t_{0}\right)}
$$

is valid for $V(X(t))>L\left(x_{0}\right), t \geq t_{0}$, where $L\left(x_{0}\right)=$ $\sup L\left(x_{0}, t\right), t \geq t_{0}$, then the system (1) is said to be globally conditional exponentially attractive or globally conditional exponentially stable in the sense of Lagrange, and $\Omega:=\{X \mid$ $\left.V(X(t)) \leq L\left(x_{0}\right), t \geq t_{0}\right\}$ is called the globally conditional exponentially attractive set.

\section{Qualitative Analysis}

We call the dynamic system (1) the first class three-dimensional chaotic system with cross-product nonlinearities (1), if there are some nonzero numbers $\left\{\lambda_{1}, \lambda_{2}, \lambda_{3}\right\}$ so as to satisfy conditions

$$
\begin{gathered}
\lambda_{1}^{2} b_{111}=0, \quad \lambda_{1}^{2}\left(b_{112}+b_{121}\right)+\lambda_{2}^{2} b_{211}=0, \\
\lambda_{2}^{2} b_{222}=0, \quad \lambda_{2}^{2}\left(b_{221}+b_{212}\right)+\lambda_{1}^{2} b_{122}=0, \\
\lambda_{3}^{2} b_{333}=0, \quad \lambda_{3}^{2}\left(b_{313}+b_{331}\right)+\lambda_{1}^{2} b_{133}=0, \\
\lambda_{1}^{2}\left(b_{113}+b_{131}\right)+\lambda_{3}^{2} b_{311}=0, \\
\lambda_{2}^{2}\left(b_{223}+b_{232}\right)+\lambda_{3}^{2} b_{322}=0, \\
\lambda_{3}^{2}\left(b_{323}+b_{332}\right)+\lambda_{2}^{2} b_{233}=0, \\
\lambda_{1}^{2}\left(b_{123}+b_{132}\right)+\lambda_{2}^{2}\left(b_{213}+b_{231}\right)+\lambda_{3}^{2}\left(b_{321}+b_{312}\right)=0 .
\end{gathered}
$$

Condition (6) is satisfied by some known three-dimensional quadratic autonomous chaotic systems, the wellknown Lorenz system [1-3], the Rössler system [5], the Rucklidge system [6], and the Chen system [7, 8]. Lorenz systems are widely studied and the references therein [912, 19-21]. For example, consider the classical Lorenz system

$$
\begin{aligned}
& \dot{x}=\sigma(y-x), \\
& \dot{y}=\rho x-\gamma y-x z, \\
& \dot{z}=x y-\beta z,
\end{aligned}
$$


and the general Lorenz systems

$$
\begin{aligned}
& \dot{x}=-a x+b y+y z, \\
& \dot{y}=c x-y-x z, \\
& \dot{z}=d y-z+x y, \\
& \dot{x}=-z, \\
& \dot{y}=-y-x^{2} \\
& \dot{z}=1.7 x+y+1.7 .
\end{aligned}
$$

Thus it can be seen that condition (6) is very important in qualitative analysis of the exponentially attractive set and positive invariant set of Lorenz systems.

We will research this dynamic system in two cases.

First, supposing $b_{111}=b_{122}=b_{133}=b_{211}=b_{222}=b_{233}=$ $b_{311}=b_{322}=b_{333}=0, b_{i j j}=-b_{i j i}, i, j=1,2,3, \exists b_{i j k} \neq-$ $b_{i k j}, i \neq j \neq k$, the dynamic system (1) can be rewritten as

$$
\begin{aligned}
& \dot{x}_{1}=\sum_{j=1}^{3} a_{1 j} x_{j}+\left(b_{123}+b_{132}\right) x_{2} x_{3}+c_{1}, \\
& \dot{x}_{2}=\sum_{j=1}^{3} a_{2 j} x_{j}+\left(b_{213}+b_{231}\right) x_{1} x_{3}+c_{2}, \\
& \dot{x}_{3}=\sum_{j=1}^{3} a_{3 j} x_{j}+\left(b_{312}+b_{321}\right) x_{1} x_{2}+c_{3} .
\end{aligned}
$$

The construction techniques of this kind of Lorenz systems are to pay attention to satisfing formula

$$
\lambda_{1}^{2}\left(b_{123}+b_{132}\right)+\lambda_{2}^{2}\left(b_{213}+b_{231}\right)+\lambda_{3}^{2}\left(b_{321}+b_{312}\right)=0
$$

where $\lambda_{i}, i=1,2,3$ are parameters and

$$
\begin{aligned}
f(\mu, X)= & \sum_{i=1}^{3} \lambda_{i}^{2}\left(a_{i i}+\mu_{i}\right) x_{i}^{2} \\
& +\sum_{i=1}^{3}\left(\lambda_{i}^{2} c_{i}+\mu_{i} d_{i} \lambda_{i}-\mu_{i} \lambda_{i}^{2} d_{i}-\sum_{j=1}^{3} \lambda_{j} d_{j} a_{j i}\right) x_{i} \\
& +\sum_{i=1}^{3}\left(\lambda_{i} d_{i} c_{i}-\lambda_{i} d_{i}^{2}\right)
\end{aligned}
$$

where $\mu_{i}, i=1,2,3$ are undetermined parameters. And we always assume that the supremum $f(\mu, X)<+\infty$ in the paper.
Lemma 6. Suppose $\lambda_{i}>0, i=1,2,3$,

$$
\begin{gathered}
\lambda_{1}^{2} a_{12}+\lambda_{2}^{2} a_{21}+\lambda_{3} d_{3}\left(b_{312}+b_{321}\right)=0 \\
\lambda_{1}^{2} a_{13}+\lambda_{3}^{2} a_{31}+\lambda_{2} d_{2}\left(b_{213}+b_{231}\right)=0 \\
\lambda_{2}^{2} a_{23}+\lambda_{3}^{2} a_{32}+\lambda_{1} d_{1}\left(b_{123}+b_{132}\right)=0 \\
a_{11}+\mu_{1}<0 \\
a_{22}+\mu_{2}<0 \\
a_{33}+\mu_{3}<0
\end{gathered}
$$

The function (12) has maximum.

Proof. Consider

$$
\begin{gathered}
f_{x_{1}}^{\prime}(\mu, X)=2\left(\lambda_{1}^{2} a_{11}+\mu_{1} \lambda_{1}^{2}\right) x_{1} \\
+\left(\lambda_{1}^{2} c_{1}+\mu_{1} d_{1} \lambda_{1}-\mu_{1} \lambda_{1}^{2} d_{1}-\lambda_{1} d_{1} a_{11}\right. \\
\left.-\lambda_{2} d_{2} a_{21}-\lambda_{3} d_{3} a_{31}\right), \\
f_{x_{2}}^{\prime}(\mu, X)=2\left(\lambda_{2}^{2} a_{22}+\mu_{2} \lambda_{2}^{2}\right) x_{2} \\
+\left(\lambda_{2}^{2} c_{2}+\mu_{2} d_{2} \lambda_{2}-\mu_{2} \lambda_{2}^{2} d_{2}-\lambda_{1} d_{1} a_{12}\right. \\
\left.-\lambda_{2} d_{2} a_{22}-\lambda_{3} d_{3} a_{32}\right), \\
f_{x_{3}}^{\prime}(\mu, X)=2\left(\lambda_{3}^{2} a_{33}+\mu_{3} \lambda_{3}^{2}\right) x_{3} \\
+\left(\lambda_{3}^{2} c_{3}+\mu_{3} d_{3} \lambda_{3}-\mu_{3} \lambda_{3}^{2} d_{3}-\lambda_{1} d_{1} a_{13}\right. \\
\left.-\lambda_{2} d_{2} a_{23}-\lambda_{3} d_{3} a_{33}\right), \\
f_{x_{1}^{2}}^{\prime \prime}(\mu, X)=2 \lambda_{1}^{2}\left(a_{11}+\mu_{1}\right), \\
f_{x_{2}^{2}}^{\prime \prime}(\mu, X)=2 \lambda_{2}^{2}\left(a_{22}+\mu_{2}\right), \\
f_{x_{3}^{2}}^{\prime \prime}(\mu, X)=2 \lambda_{3}^{2}\left(a_{33}+\mu_{3}\right), \\
f_{x_{1} x_{2}}^{\prime \prime}(\mu, X)=f_{x_{2} x_{1}}^{\prime \prime}(\mu, X)=f_{x_{1} x_{3}}^{\prime \prime}(\mu, X) \\
=f_{x_{2} x_{3}}^{\prime \prime}(\mu, X)=f_{x_{3} x_{2}}^{\prime \prime}(\mu, X)=0 .
\end{gathered}
$$

Thus the Hesse matrix $H_{f}$ of the $f(\mu, X)$ is a negative definite matrix; furthermore $\max _{X \in R^{3}} f(\mu, X)$ exists.

These parameters $d_{i}, \mu_{i}, \lambda_{i}, i=1,2,3$ will be determined by solving the maximum of $f_{1}(\mu, X)$ and formula (12), and let

$$
M^{+}=\left\{\begin{array}{l}
M, \quad M>0 \\
0, \quad M \leq 0
\end{array}\right.
$$

Theorem 7. If condition (6) exists, $\eta=\min \left\{\mu_{1}, \mu_{2}, \mu_{3}\right\}, M=$ $\max _{X \in R^{3}} f(\mu, X), \lambda_{i}>0, i=1,2,3$, then the estimation

$$
\left[V(X(t))-\frac{1}{2 \eta} M^{+}\right] \leq\left[V\left(X\left(t_{0}\right)\right)-\frac{1}{2 \eta} M^{+}\right] e^{-2 \eta\left(t-t_{0}\right)}
$$


holds, and the set

$$
\begin{aligned}
\Omega= & \left\{X \mid V(X(t)) \leq \frac{1}{2 \eta} M^{+}\right\} \\
= & \left\{X \mid\left[\left(\lambda_{1} x_{1}-d_{1}\right)^{2}+\left(\lambda_{2} x_{2}-d_{2}\right)^{2}+\left(\lambda_{3} x_{3}-d_{3}\right)^{2}\right]\right. \\
& \left.\leq \frac{1}{\eta} M^{+}\right\}
\end{aligned}
$$

is the globally exponentially attractive set and positive invariant set of system (10); that is,

$$
\varlimsup_{t \rightarrow \infty} V(X(t)) \leq \frac{1}{2 \eta} M^{+}
$$

Proof. Differentiating the Lyapunov function $V(X(t))$ in (3) with respect to time $t$ along the trajectory of system (10) yields

$$
\begin{aligned}
\left.\dot{V}(X(t))\right|_{(10)}= & \lambda_{1}\left(\lambda_{1} x_{1}-d_{1}\right) \dot{x}_{1}+\lambda_{2}\left(\lambda_{2} x_{2}-d_{2}\right) \dot{x}_{2} \\
& +\lambda_{3}\left(\lambda_{3} x_{3}-d_{3}\right) \dot{x}_{3} \\
= & -\sum_{j=1}^{3} \mu_{j}\left(\lambda_{j} x_{j}-d_{j}\right)^{2}+\lambda_{1}\left(\lambda_{1} x_{1}-d_{1}\right) \\
& \times\left(\sum_{j=1}^{3} a_{1 j} x_{j}+\mu_{1} \lambda_{1}^{-1}\left(\lambda_{1} x_{1}-d_{1}\right)\right. \\
& \left.+\left(b_{123}+b_{132}\right) x_{2} x_{3}+c_{1}\right) \\
& +\lambda_{2}\left(\lambda_{2} x_{2}-d_{2}\right) \\
& \times\left(\sum_{j=1}^{3} a_{2 j} x_{j}+\mu_{2} \lambda_{2}^{-1}\left(\lambda_{2} x_{2}-d_{2}\right)\right. \\
& +\sum_{j=1}^{3}\left(\lambda_{j} x_{j}-d_{j}\right)^{2}+f(\mu, X) \\
& +\lambda_{3}\left(\lambda_{3} x_{3}-d_{3}\right) \\
& +\left(b_{3 j} x_{j}+\mu_{3} \lambda_{3}^{-1}\left(\lambda_{3} x_{3}-d_{3}\right)\right. \\
& \left.+\left(b_{312}+b_{321}\right) x_{1} x_{2}+c_{3}\right) \\
& \\
& \\
& \left.+c_{2}\right)
\end{aligned}
$$

$$
\begin{aligned}
& \leq-\eta \sum_{j=1}^{3}\left(\lambda_{j} x_{j}-d_{j}\right)^{2}+M^{+} \\
& \leq-2 \eta\left[V(X(t))-\frac{1}{2 \eta} M^{+}\right] \leq 0, \\
& \text { when } V(X(t))>\frac{1}{2 \eta} M^{+},
\end{aligned}
$$

where $\mu_{j}>0, j=1,2,3$. Integrating both sides of (19) yields (16) and (17). By the definition, taking into account limit on both sides of the above inequality (16) as $t \rightarrow+\infty$ results in inequality (18).

Now, the characters of some of the chaotic systems known are analysed by condition (6). When $a_{11}=-\sigma, a_{12}=\sigma, a_{21}=$ $\rho, a_{22}=-\gamma, a_{33}=-\beta, b_{213}=-1, b_{312}=1$, else $a_{i j}=0, b_{i j k}=$ $0, c_{1}=c_{2}=c_{3}=0$, and $\lambda_{1}=\sqrt{\lambda}, \lambda_{2}=\lambda_{3}=1, d_{1}=d_{2}=$ $0, d_{3}=\lambda \sigma+\rho, \mu_{1}=\sigma, \mu_{2}=\gamma, \mu_{3}=\min \{\sigma, \gamma\}, \eta=\eta_{1}=\mu_{3}$, $\beta>\eta_{1}$, system (10) can be rewritten as system (7):

$$
\begin{aligned}
V(X(t)) & =\frac{1}{2}\left[\lambda x_{1}^{2}+x_{2}^{2}+\left(x_{3}-\lambda \sigma-\rho\right)^{2}\right] \\
f(\mu, X)= & -\left(\beta-\eta_{1}\right) x_{3}^{2}+\left(\beta-2 \eta_{1}\right)(\lambda \sigma+\rho) x_{3} \\
& +\eta_{1}(\lambda \sigma+\rho)^{2} .
\end{aligned}
$$

We have $M=\beta^{2}(\lambda \sigma+\rho)^{2} / 4\left(\beta-\eta_{1}\right)$. Thus

$$
\begin{gathered}
{\left[V(X(t))-\frac{\beta^{2}(\lambda \sigma+\rho)^{2}}{8\left(\beta-\eta_{1}\right) \eta_{1}}\right]} \\
\quad \leq\left[V\left(X\left(t_{0}\right)\right)-\frac{\beta^{2}(\lambda \sigma+\rho)^{2}}{8\left(\beta-\eta_{1}\right) \eta_{1}}\right] e^{-2 \eta_{1}\left(t-t_{0}\right)}, \\
\Omega_{1}=\left\{X \mid V(X) \leq \frac{\beta^{2}(\lambda \sigma+\rho)^{2}}{8\left(\beta-\eta_{1}\right) \eta_{1}}\right\} \\
\quad=\left\{X \mid \lambda x_{1}^{2}+x_{2}^{2}+\left(x_{3}-\lambda \sigma-\rho\right)^{2} \leq \frac{\beta^{2}(\lambda \sigma+\rho)^{2}}{4\left(\beta-\eta_{1}\right) \eta_{1}}\right\}
\end{gathered}
$$

is the globally exponentially attractive set and positive invariant set of system (7).

Example 8. Further, taking ito accout $\mu_{1}=\sigma, \mu_{2}=\gamma, \mu_{3}=$ $\beta / 2, \eta=\eta_{2}=\min \{\sigma, \gamma, \beta / 2\}$, the estimate

$$
\begin{aligned}
& {\left[V(X(t))-\frac{\beta(\lambda \sigma+\rho)^{2}}{4 \eta_{2}}\right]} \\
& \quad \leq\left[V\left(X\left(t_{0}\right)\right)-\frac{\beta(\lambda \sigma+\rho)^{2}}{4 \eta_{2}}\right] e^{-2 \eta_{2}\left(t-t_{0}\right)}
\end{aligned}
$$


holds and that

$$
\begin{aligned}
\Omega_{2} & =\left\{X \mid V(X) \leq \frac{\beta(\lambda \sigma+\rho)^{2}}{4 \eta_{2}}\right\} \\
& =\left\{X \mid \lambda x_{1}^{2}+x_{2}^{2}+\left(x_{3}-\lambda \sigma-\rho\right)^{2} \leq \frac{\beta(\lambda \sigma+\rho)^{2}}{2 \eta_{2}}\right\}
\end{aligned}
$$

is the globally uniform exponentially attractive set and positive invariant set of system (7).

Proof. Again applying Lyapunov function given in (19) and evaluating the derivative of $d V_{1} / d t$ along the trajectory of system (16) lead to

$$
\begin{gathered}
f(\mu, X)=-\frac{x_{3}^{2} \beta}{2}+\frac{(\lambda \sigma+\rho)^{2} \beta}{2}, \\
M=\frac{\beta(\lambda \sigma+\rho)^{2}}{2} .
\end{gathered}
$$

The conclusion of Example 9 is obtained.

Example 9. Furthermore, choose $\mu_{1}=\sigma, \mu_{2}=\gamma, \mu_{3}=\beta, 0<$ $\xi_{1}<\beta, \eta=\eta_{3}=\min \left\{\sigma, \gamma, \xi_{1}\right\}$. Get

$$
\begin{aligned}
f(\mu, X)= & -\left(\beta-\eta_{3}\right) x_{3}^{2}+\left(\beta-2 \eta_{3}\right)(\lambda \sigma+\rho) x_{3} \\
& +\eta_{2}(\lambda \sigma+\rho)^{2}, \\
M= & \frac{\beta^{2}(\lambda \sigma+\rho)^{2}}{4\left(\beta-\eta_{3}\right)} .
\end{aligned}
$$

Then, the estimate

$$
\begin{aligned}
& {\left[V(X(t))-\frac{\beta^{2}(\lambda \sigma+\rho)^{2}}{8\left(\beta-\eta_{3}\right) \eta_{3}}\right]} \\
& \quad \leq\left[V\left(X\left(t_{0}\right)\right)-\frac{\beta^{2}(\lambda \sigma+\rho)^{2}}{8\left(\beta-\eta_{3}\right) \eta_{3}}\right] e^{-2 \eta_{3}\left(t-t_{0}\right)}
\end{aligned}
$$

holds and that

$$
\begin{aligned}
\Omega_{3} & =\left\{X \mid V(X) \leq \frac{\beta^{2}(\lambda \sigma+\rho)^{2}}{8\left(\beta-\eta_{3}\right) \eta_{3}}\right\} \\
& =\left\{X \mid \lambda x_{1}^{2}+x_{2}^{2}+\left(x_{3}-\lambda \sigma-\rho\right)^{2} \leq \frac{\beta^{2}(\lambda \sigma+\rho)^{2}}{4\left(\beta-\eta_{3}\right) \eta_{3}}\right\}
\end{aligned}
$$

is the globally exponentially attractive set and positive invariant set of system (7).

Example 10. Taking $a_{11}=-a, a_{12}=b, a_{21}=c, a_{22}=-1$, $a_{32}=d, a_{33}=-1, b_{123}=b_{312}=1, b_{213}=-1$ else $a_{i j}=0$, $b_{i j k}=0, c_{1}=c_{2}=c_{3}=0$, and $\lambda_{1}=\lambda_{3}=1, \lambda_{2}=\sqrt{2}, d_{1}=d$, $d_{2}=0, d_{3}=b+2 c$, system $(6), V(X(t))$, and $f(u, X)$ can be rewritten as system (8):

$$
\begin{aligned}
V(X(t)) & =\frac{1}{2}\left[\left(x_{1}-d\right)^{2}+2 x_{2}^{2}+\left(x_{3}-b-2 c\right)^{2}\right] \\
f(\mu, X)= & -\left(a-\mu_{1}\right) x_{1}^{2}+\left(a-2 \mu_{1}\right) d x_{1}-2\left(1-\mu_{2}\right) x_{2}^{2} \\
& -2(b+c) d x_{2}-\left(1-\mu_{3}\right) x_{3}^{2} \\
& +(b+2 c)\left(1-2 \mu_{3}\right) x_{3}+\mu_{1} d^{2}+\mu_{3}(b+2 c)^{2} .
\end{aligned}
$$

Thus

$$
\begin{aligned}
M= & \frac{\left(a-2 \mu_{1}\right)^{2} d^{2}}{4\left(a-\mu_{1}\right)}+\frac{(b+c)^{2} d^{2}}{2\left(1-\mu_{2}\right)}+\frac{(b+2 c)^{2}\left(1-2 \mu_{3}\right)}{4\left(1-\mu_{3}\right)} \\
& +\mu_{1} d^{2}+\mu_{3}(b+2 c)^{2} .
\end{aligned}
$$

We have

$$
V(x(t))-M \leq\left[V\left(x\left(t_{0}\right)\right)-M\right] e^{-2 \eta\left(t-t_{0}\right)},
$$

then

$$
\begin{aligned}
\Omega_{4} & =\{X \mid V(x(t))-M\} \\
& =\left\{X \mid\left(x_{1}-d\right)^{2}+2 x_{2}^{2}+\left(x_{3}-b-2 c\right)^{2} \leq 2 M\right\}
\end{aligned}
$$

is the estimation of the globally exponentially attractive and positive invariant sets of system (8).

If $b_{111}=b_{222}=b_{333}=0, \exists b_{i j j} \neq 0, i, j=1,2,3$, the dynamic system (1) is shown as

$$
\begin{aligned}
& \dot{x}_{1}=\sum_{j=1}^{3} a_{1 j} x_{j}+\sum_{i=2,3} b_{1 i i} x_{i}^{2}+\sum_{i \neq j=1}^{3}\left(b_{1 i j}+b_{1 j i}\right) x_{i} x_{j}+c_{1}, \\
& c \dot{x}_{2}=\sum_{j=1}^{3} a_{2 j} x_{j}+\sum_{i=1,3} b_{2 i i} x_{i}^{2}+\sum_{i \neq j=1}^{3}\left(b_{2 i j}+b_{2 j i}\right) x_{i} x_{j}+c_{2}, \\
& \dot{x}_{3}=\sum_{j=1}^{3} a_{3 j} x_{j}+\sum_{i=1,2} b_{3 i i} x_{i}^{2}+\sum_{i \neq j=1}^{3}\left(b_{3 i j}+b_{3 j i}\right) x_{i} x_{j}+c_{3} .
\end{aligned}
$$

In this case, we can take into account

$$
\begin{gathered}
f(\mu, X)=\sum_{i=1}^{3}\left[\lambda_{i}^{2}\left(a_{i i}+\mu_{i}\right)+\lambda_{[i+1]_{3}} d_{[i+1]_{3}} b_{[i+1]_{3}, i i}\right. \\
\left.+\lambda_{[i+2]_{3}} d_{[i+2]_{3}} b_{[i+2]_{3}, i i}\right] x_{i}^{2}
\end{gathered}
$$




$$
\begin{aligned}
& +\sum_{i<j, 1}^{3}\left(\lambda_{i}^{2} a_{i j}+\lambda_{j}^{2} a_{j i}+\lambda_{1} d_{1}\left(b_{1 i j}+b_{1 j i}\right)\right. \\
& \left.\quad+\lambda_{2} d_{2}\left(b_{2 i j}+b_{2 j i}\right)+\lambda_{3} d_{3}\left(b_{3 i j}+b_{3 j i}\right)\right) x_{i} x_{j} \\
& +\sum_{i=1}^{3}\left(\lambda_{i}^{2}\left(c_{i}-\mu_{i} d_{i}\right)+\mu_{i} \lambda_{i} d_{i}-a_{1 i} \lambda_{1} d_{1}\right. \\
& \left.\quad-a_{2 i} \lambda_{2} d_{2}-a_{3 i} \lambda_{3} d_{3}\right) x_{1} \\
& +\sum_{i=1}^{3} \lambda_{i} d_{i}\left(c_{i}-d_{i}\right)
\end{aligned}
$$

where $[\cdot]_{3}$ denotes modulo-3.

Theorem 11. Suppose that $G_{0}=\left(x_{1}^{0}, x_{2}^{0}, \ldots, x_{n}^{0}\right)$ is the stable point of the $f(\mu, X)$ defined by (33). If the Hesse matrix of the $f(\mu, X)$ is a negative definite matrix, the $f(\mu, X)$ has maximum Mand the estimation

$$
\begin{aligned}
& {\left[V(X(t))-\frac{1}{2 \eta} M^{+}\right]} \\
& \quad \leq\left[V\left(X\left(t_{0}\right)\right)-\frac{1}{2 \eta} M^{+}\right] e^{-2 \eta\left(t-t_{0}\right)}
\end{aligned}
$$

holds; that is,

$$
\varlimsup_{t \rightarrow \infty} V(X(t)) \leq \frac{1}{2 \eta} M^{+}
$$

and the set

$$
\begin{aligned}
\Omega & =\left\{X \mid V(X(t)) \leq \frac{1}{2 \eta} M^{+}\right\} \\
= & \left\{X \mid\left[\left(\lambda_{1} x_{1}-d_{1}\right)^{2}+\left(\lambda_{2} x_{2}-d_{2}\right)^{2}+\left(\lambda_{3} x_{3}-d_{3}\right)^{2}\right]\right. \\
& \left.\leq \frac{1}{\eta} M^{+}\right\}
\end{aligned}
$$

is the globally exponentially attractive set and positive invariant set of system (32).

Proof. If $G_{0}$ is the stable point of the $f(\mu, X)$, that is,

$$
\nabla(f)_{G_{0}}=\left(f_{x_{1}}^{\prime}, f_{x_{2}}^{\prime}, f_{x_{3}}^{\prime}\right)=0
$$

and the Hesse matrix $H_{f}$ of the $f(\mu, X)$ is a negative definite matrix, namely,

$$
\begin{gathered}
f_{x_{1} x_{1}}^{\prime \prime}<0, \quad\left|\begin{array}{ll}
f_{x_{1} x_{1}}^{\prime \prime} & f_{x_{1} x_{2}}^{\prime \prime} \\
f_{x_{2} x_{1}}^{\prime \prime} & f_{x_{2} x_{2}}^{\prime \prime}
\end{array}\right|>0, \\
\left|\begin{array}{lll}
f_{x_{1} x_{1}}^{\prime \prime} & f_{x_{1} x_{2}}^{\prime \prime} & f_{x_{1} x_{3}}^{\prime \prime} \\
f_{x_{2} x_{1}}^{\prime \prime} & f_{x_{2} x_{2}}^{\prime \prime} & f_{x_{2} x_{3}}^{\prime \prime} \\
f_{x_{3} x_{1}}^{\prime \prime} & f_{x_{3} x_{2}}^{\prime \prime} & f_{x_{3} x_{3}}^{\prime \prime}
\end{array}\right|<0 .
\end{gathered}
$$

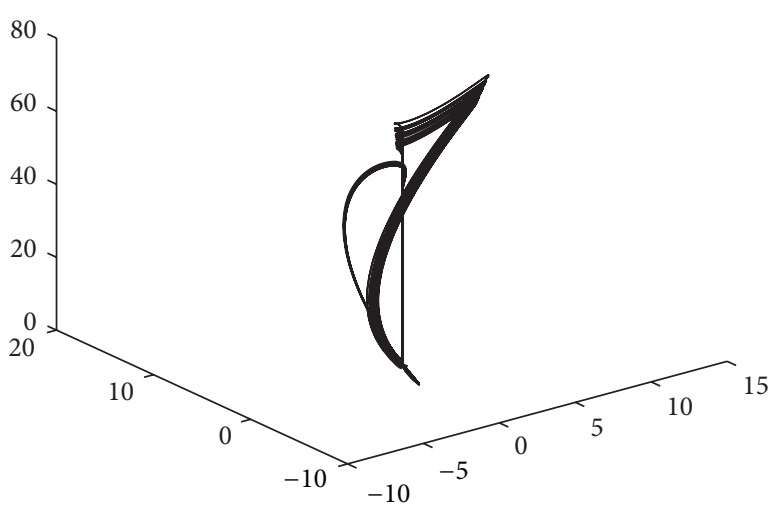

Figure 1: Simulation of system (40).

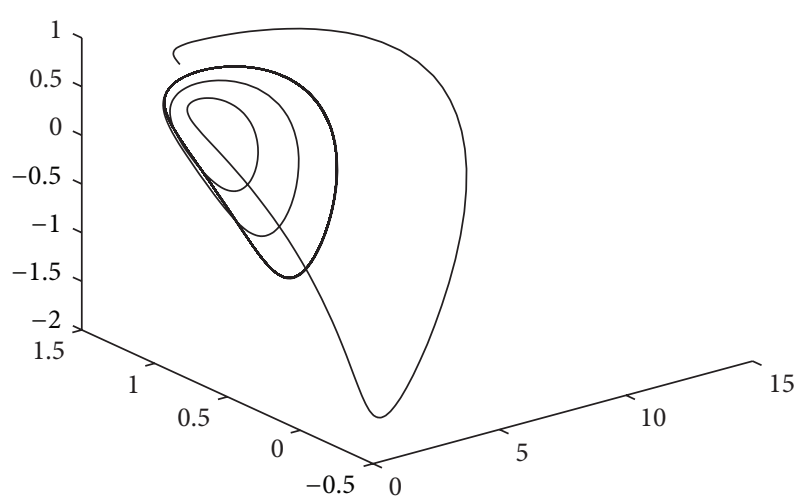

FIGURE 2: Simulation of system (43).

The $f(\mu, X)$ has the maximum $M$. Differentiating the Lyapunov function $V(X(t))$ in (3) with respect to time $t$ along the trajectory of system (32) yields

$$
\begin{aligned}
&\left.\dot{V}(X(t))\right|_{(32)}= \lambda_{1}\left(\lambda_{1} x_{1}-d_{1}\right) \dot{x}_{1}+\lambda_{2}\left(\lambda_{2} x_{2}-d_{2}\right) \dot{x}_{2} \\
&+\lambda_{3}\left(\lambda_{3} x_{3}-d_{3}\right) \dot{x}_{3} \\
& \leq-\eta \sum_{j=1}^{3}\left(\lambda_{j} x_{j}-d_{j}\right)^{2}+f(\mu, X) \\
& \leq-\eta \sum_{j=1}^{3}\left(\lambda_{j} x_{j}-d_{j}\right)^{2}+M^{+} \\
& \leq-2 \eta\left[V(X(t))-\frac{1}{2 \eta} M^{+}\right] \leq 0 \\
& \text { when } V(X(t))>\frac{1}{2 \eta} M^{+} .
\end{aligned}
$$

The proof is complete.

\section{Switched Chaotic Systems}

Condition (6) has helpfully provided us with instructions on how to find the new chaotic systems. We construct a series 


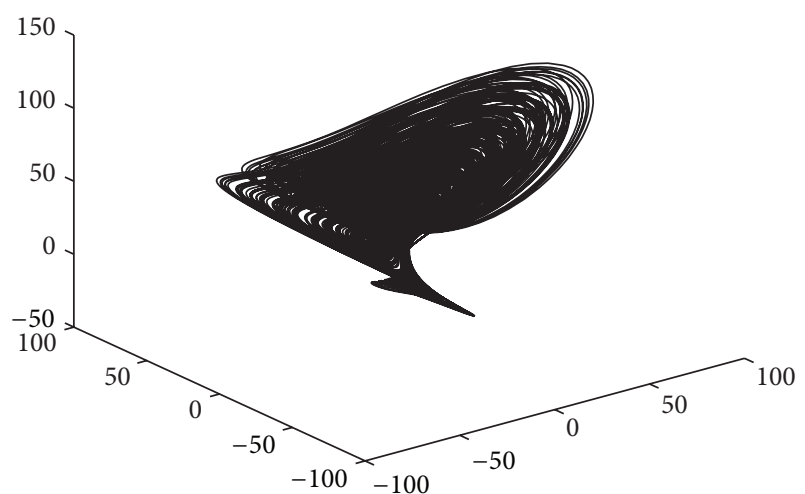

FIGURE 3: Simulation of system (44).

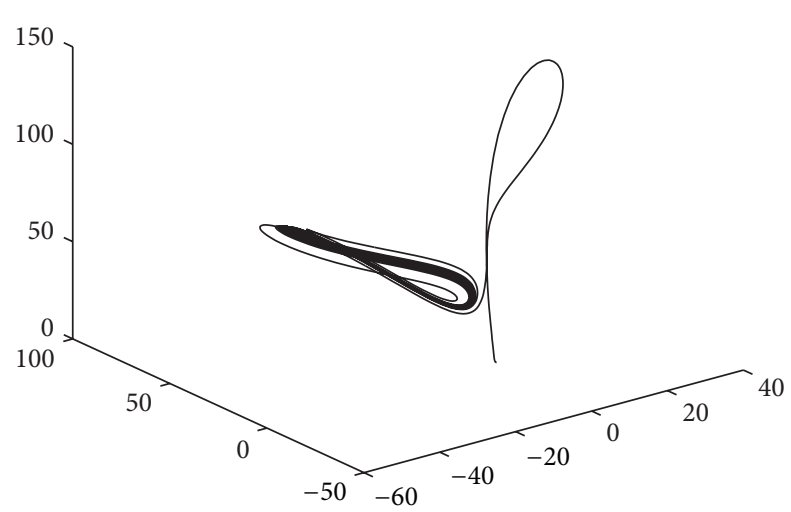

FIGURE 4: Simulation of system (45).

of new chaotic systems that the condition (6) is fulfilled and study the switching system between them.

Example 12. Consider a Lorenz system shown in Figure 1:

$$
\begin{aligned}
& \dot{x}_{1}=-12 x_{1}+5 x_{2}-0.8 x_{1} x_{3}+x_{2} x_{3}, \\
& \dot{x}_{2}=28 x_{1}-x_{2}-x_{1} x_{3}, \\
& \dot{x}_{3}=-3 x_{2}-x_{3}+10 x_{1}^{2}+x_{1} x_{2} .
\end{aligned}
$$

Solution. Here

$$
\begin{gathered}
a_{11}=-12, \quad a_{12}=5, \quad a_{21}=28, \quad a_{22}=-1, \quad a_{32}=-3, \\
a_{33}=-1, \quad \text { els } a_{i j}=0, \quad b_{113}=b_{131}=-0.4, \\
b_{123}=b_{132}=0.5, \quad b_{213}=b_{231}=-0.5, \quad b_{311}=10, \\
b_{312}=b_{321}=0.5, \quad \text { els } b_{i j k}=0, \quad \lambda_{1}=\sqrt{12.5}, \\
\lambda_{2}=\sqrt{13.5}, \quad \lambda_{3}=1, \quad d_{1}=-\frac{6}{25}, \quad d_{2}=\frac{4}{45}, \\
d_{3}=440.5, \quad \mu_{1}=2, \quad \mu_{2}=\mu_{3}=\frac{1}{2}, \quad \eta=\frac{1}{2}, \\
f_{2}(\mu, X)=-125 x_{1}^{2}-6.75 x_{2}^{2}-0.5 x_{3}^{2}
\end{gathered}
$$

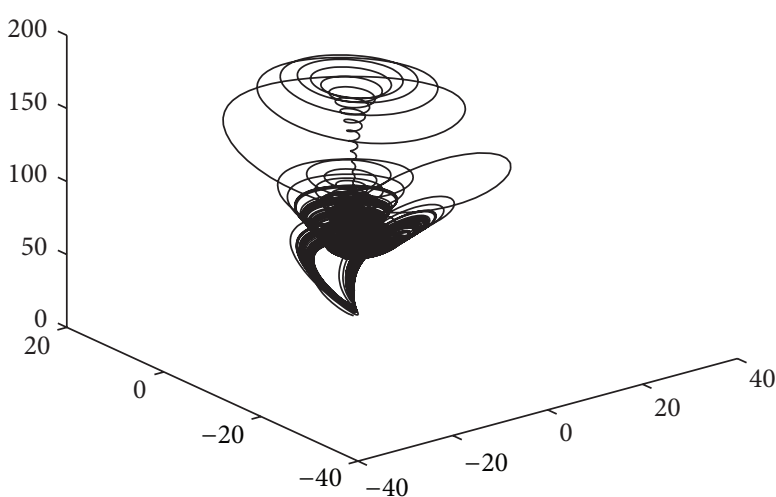

FIgURE 5: Simulation of system (46).

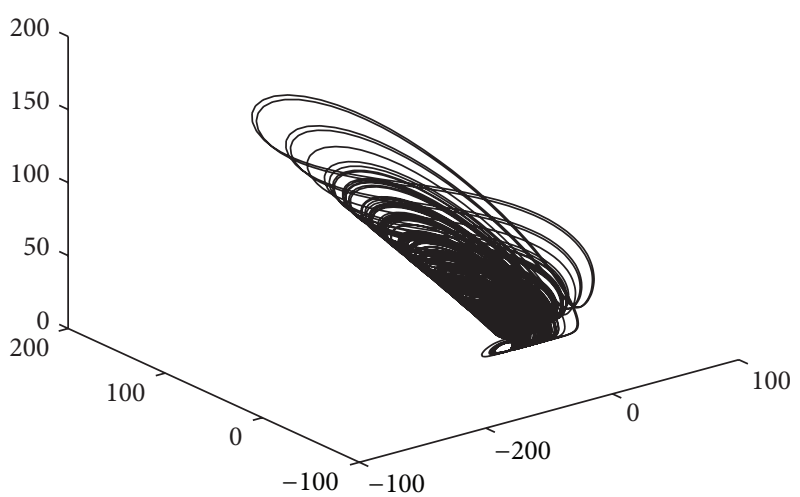

FIGURE 6: Simulation of system (47).

$$
\begin{gathered}
+\left(-\frac{48}{25} \sqrt{12.5}-\frac{112}{45} \sqrt{13.5}\right) x_{1} \\
+\left(\frac{168}{25} \sqrt{12.5}+\frac{2643}{2}\right) x_{2}+\frac{39293196661}{405000},
\end{gathered}
$$$$
f_{2 x_{1}}^{\prime}(\mu, X)=-250 x_{1}-\left(\frac{48}{25} \sqrt{12.5}+\frac{112}{45} \sqrt{13.5}\right),
$$$$
f_{2 x_{1}}^{\prime}(\mu, X)=0, \quad x_{1}=-\frac{1}{1250}\left(\frac{48}{5} \sqrt{12.5}+\frac{112}{9} \sqrt{13.5}\right)
$$$$
\approx 0.064 \text {, }
$$

$$
f_{2 x_{2}}^{\prime}(\mu, X)=-13.5 x_{2}+\left(\frac{168}{25} \sqrt{12.5}+\frac{2643}{2}\right) \text {, }
$$

$f_{2 x_{2}}^{\prime}(\mu, X)=0, \quad x_{2}=\frac{30}{135}\left(\frac{56}{25} \sqrt{12.5}+\frac{881}{2}\right) \approx 99.65$,

$f_{2 x_{3}}^{\prime}(\mu, X)=-x_{3}, \quad f_{2 x_{3}}^{\prime}(\mu, X)=0, \quad x_{3}=0$,

$f_{2 x_{1}^{2}}^{\prime \prime}(\mu, X)=-250, \quad f_{2 x_{2}^{2}}^{\prime \prime}(\mu, X)=-13.5$,

$$
f_{2 x_{3}^{2}}^{\prime \prime}(\mu, X)=-1,
$$

$f_{2 x_{1} x_{2}}^{\prime \prime}(\mu, X)=f_{2 x_{2} x_{1}}^{\prime \prime}(\mu, X)=f_{2 x_{1} x_{3}}^{\prime \prime}(\mu, X)=0$,

$f_{2 x_{3} x_{1}}^{\prime \prime}(\mu, X)=f_{2 x_{2} x_{3}}^{\prime \prime}(\mu, X)=f_{2 x_{3} x_{2}}^{\prime \prime}(\mu, X)=0$. 


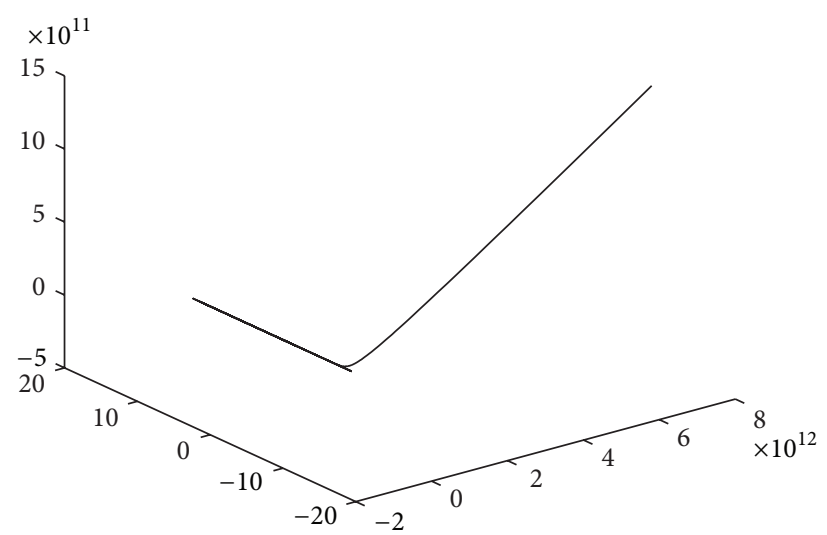

(a) System (40)-(43)

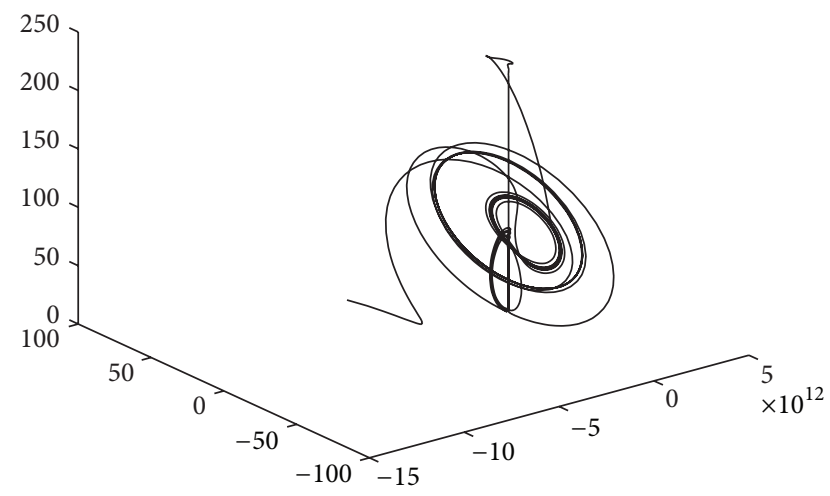

(c) System (40)-(45)

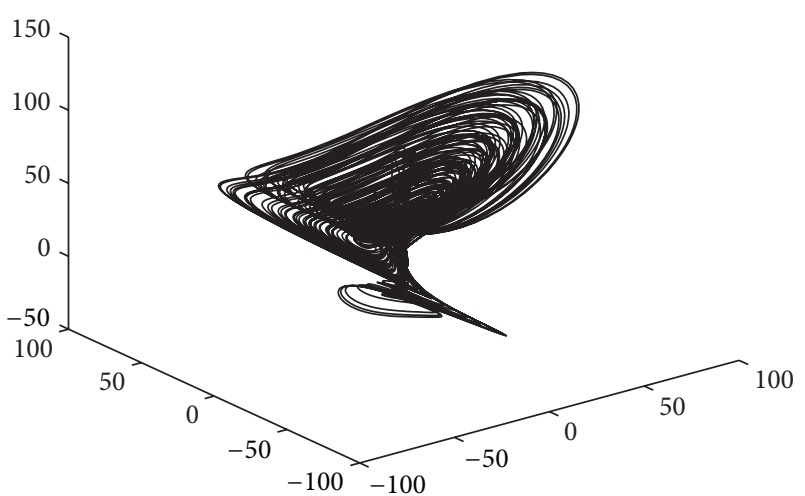

(b) System (40)-(44)

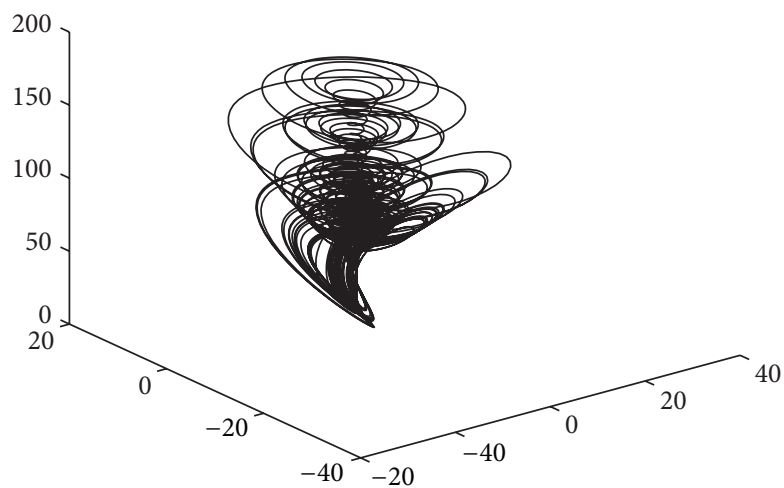

(d) System (40)-(46)

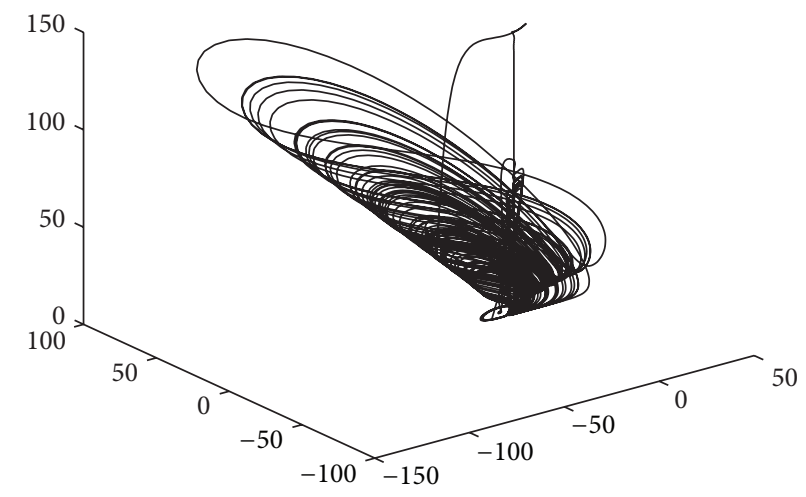

(e) System (40)-(47)

FIgURE 7: Switched system between system (40) and others.

The Hesse matrix of the $f(\mu, X)$ is a negative definite matrix, $\max f(\mu, X) \approx 164045.42$. The set

$$
\begin{gathered}
\Omega=\left\{X \mid\left(\sqrt{12.5} x_{1}+\frac{5}{26}\right)^{2}+\left(\sqrt{13.5} x_{2}-\frac{4}{45}\right)^{2}\right. \\
\left.+\left(x_{3}-440.5\right)^{2} \leq 328090.84\right\}
\end{gathered}
$$

is the globally exponentially attractive set and positive invariant set of system (40).
Note. (a) If the Hesse matrix of the $f(\mu, X)$ is not a negative definite matrix, the $f(\mu, X)$ has no maximum $M$.

(b) If $\exists a_{i_{0} i_{0}} \geq 0, \lim _{x_{i_{0}} \rightarrow \infty} f(\mu, X)=+\infty$, this type of chaotic system needs further research.

(c) We call the dynamic system (1) the second class threedimensional chaotic system with cross-product nonlinearities, if it does not satisfy condition (6). For this class of chaotic systems, $f(\mu, X)$ is a cubic polynomial and there is not maximum if we choose energy function (3) differentiating this Lyapunov function with respect to $t$ along the trajectory of system (1). It is very useful to research these problems. 


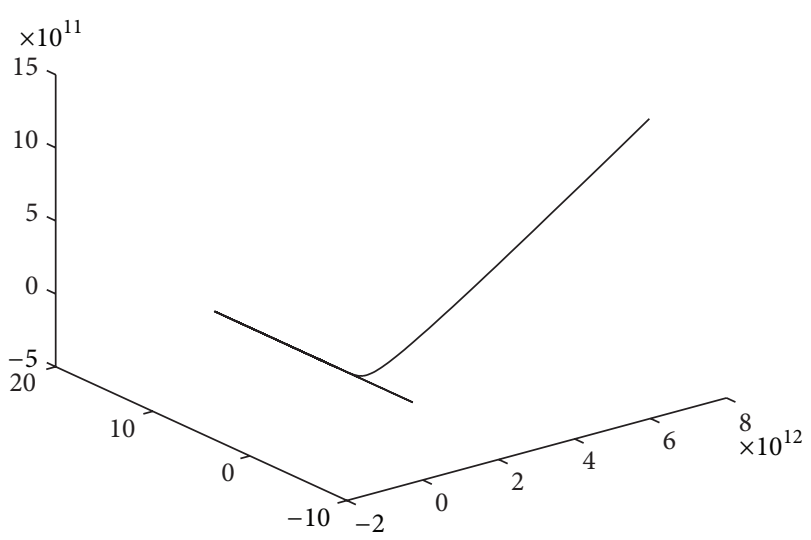

(a) System (43)-(40)

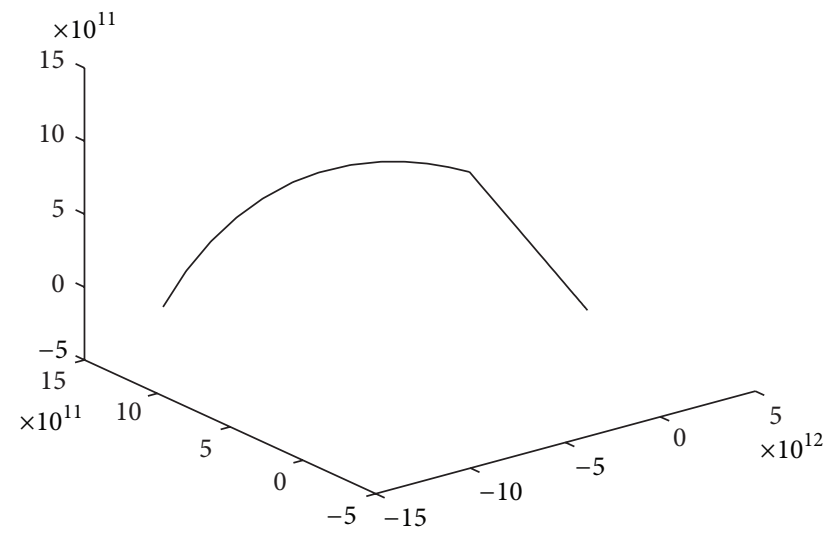

(c) System (43)-(45)

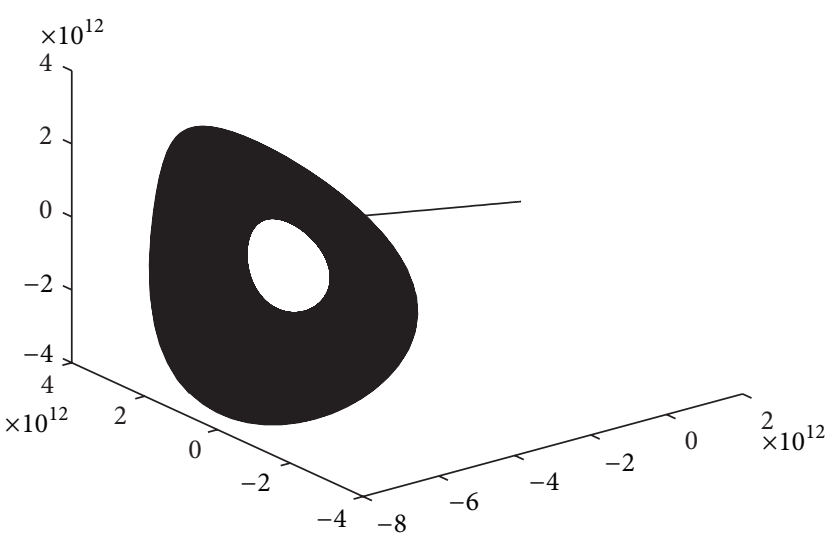

(b) System (43)-(44)

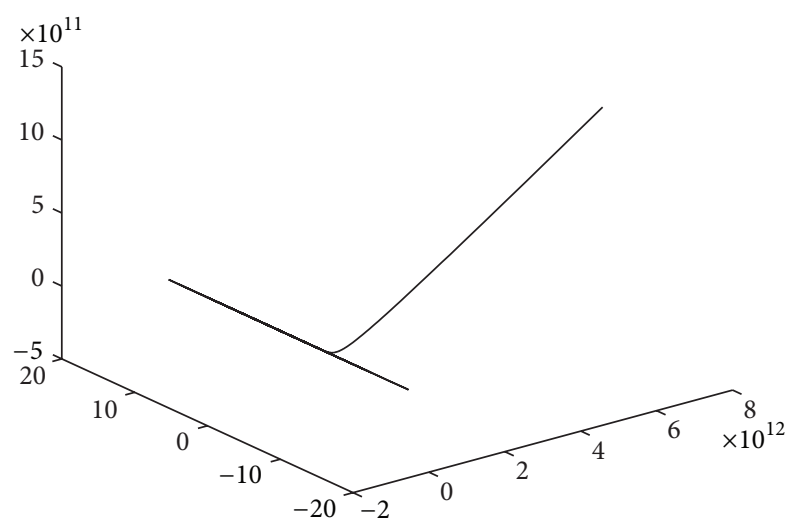

(d) System (43)-(46)

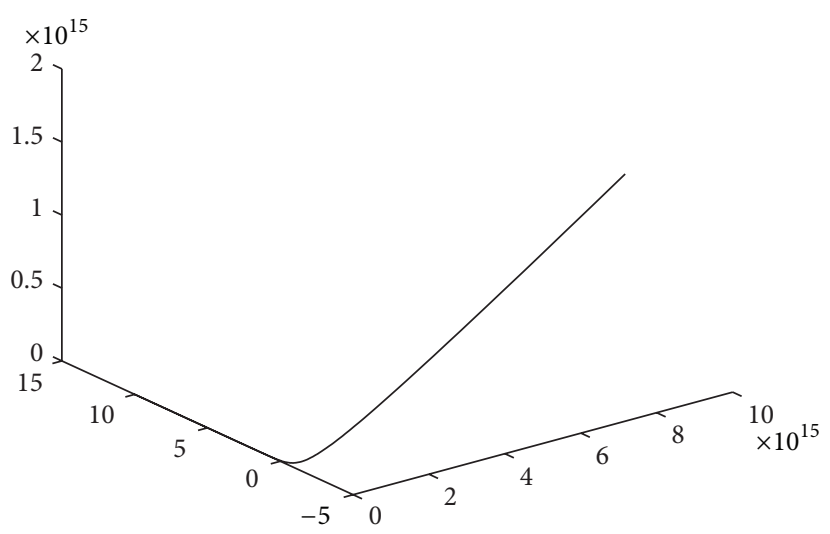

(e) System (43)-(47)

FIGURE 8: Switched system between system (43) and others.

Example 13. The new chaotic system shown in Figure 2 is

$$
\begin{aligned}
& \dot{x}_{1}=-2 x_{1}+5 x_{3}+5.7 x_{1} x_{3}+4.7 x_{2} x_{3}+4 \\
& \dot{x}_{2}=-x_{1}-2 x_{2}+3 x_{3}+5 \\
& \dot{x}_{3}=-6 x_{1}-2 x_{2}-4 x_{3}+0.2 x_{1}^{2}+3.4 x_{1} x_{2}+7 .
\end{aligned}
$$

Example 14. The chaotic system shown in Figure 3 is

$$
\begin{aligned}
& \dot{x}_{1}=-11 x_{1}+0.15 x_{1} x_{2}+1.38 x_{2} x_{3}+1, \\
& \dot{x}_{2}=30 x_{1}-x_{2}-x_{1} x_{3}+0.1 x_{2} x_{3}, \\
& \dot{x}_{3}=15 x_{2}-2.5 x_{3}+x_{1} x_{2} .
\end{aligned}
$$




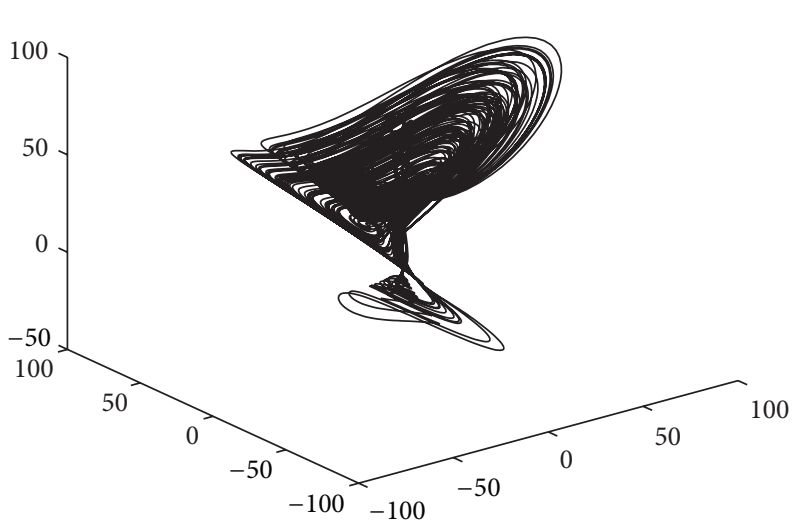

(a) System (44)-(40)

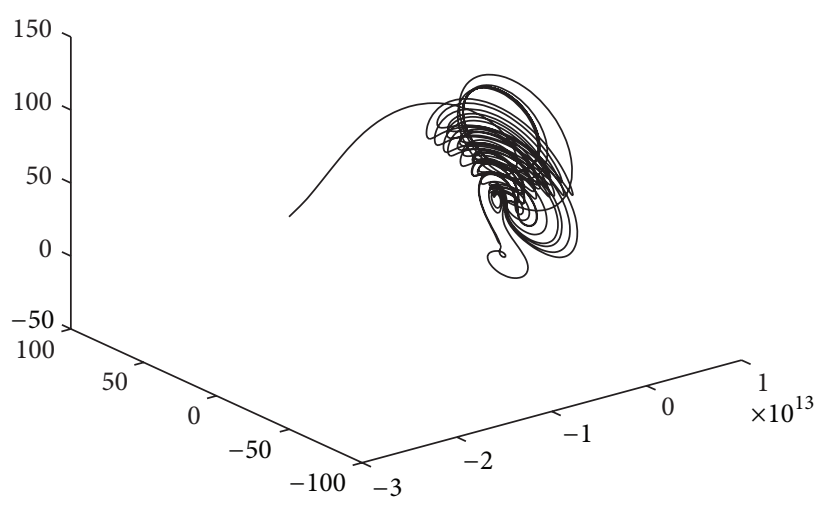

(c) System (44)-(45)

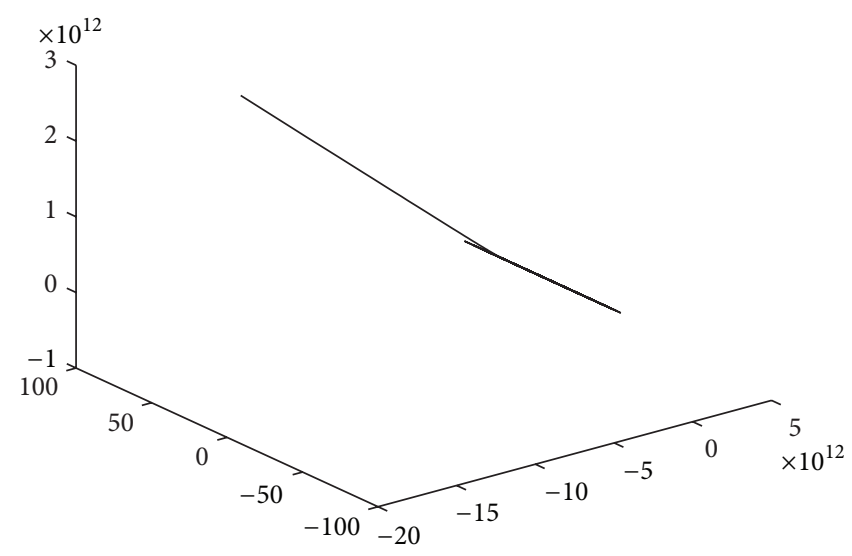

(b) System (44)-(43)

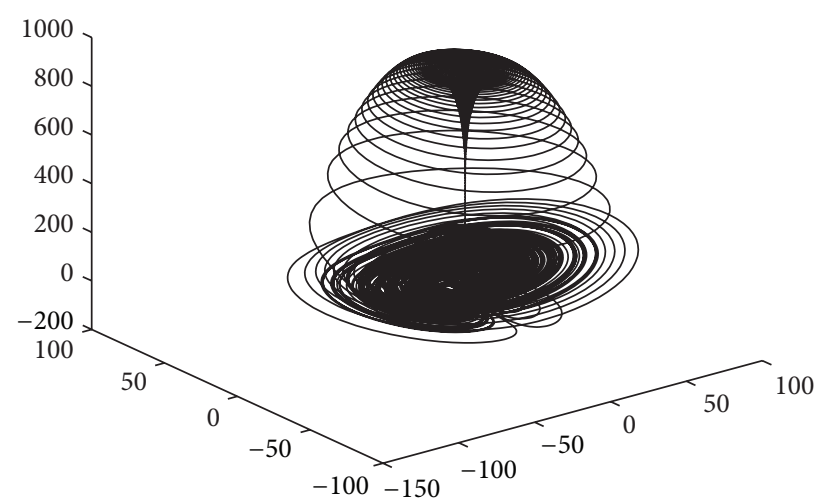

(d) System (44)-(46)

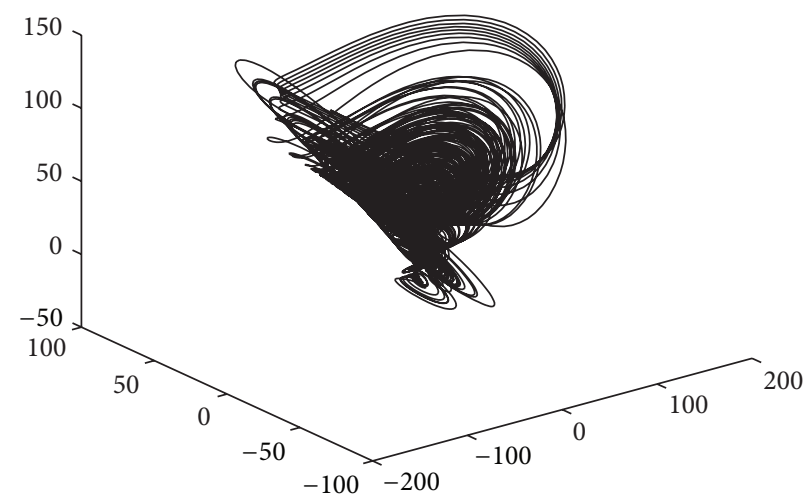

(e) System (44)-(47)

Figure 9: Switched system between Example (44) and others.

Example 15. The chaotic system shown in Figure 4 is

$$
\begin{aligned}
& \dot{x}_{1}=30\left(x_{2}-x_{1}\right)-0.48 x_{1}^{2}, \\
& \dot{x}_{2}=80 x_{1}-6 x_{2}-x_{1} x_{3}, \\
& \dot{x}_{3}=x_{1} x_{2}-5 x_{3} .
\end{aligned}
$$

Example 16. The chaotic system shown in Figure 5 is

$$
\begin{aligned}
& \dot{x}_{1}=-12 x_{1}+5 x_{2}+x_{2} x_{3}, \\
& \dot{x}_{2}=28 x_{1}-x_{2}-x_{1} x_{3}, \\
& \dot{x}_{3}=-3 x_{2}-x_{3}+4 x_{1}^{2}+x_{1} x_{2} .
\end{aligned}
$$




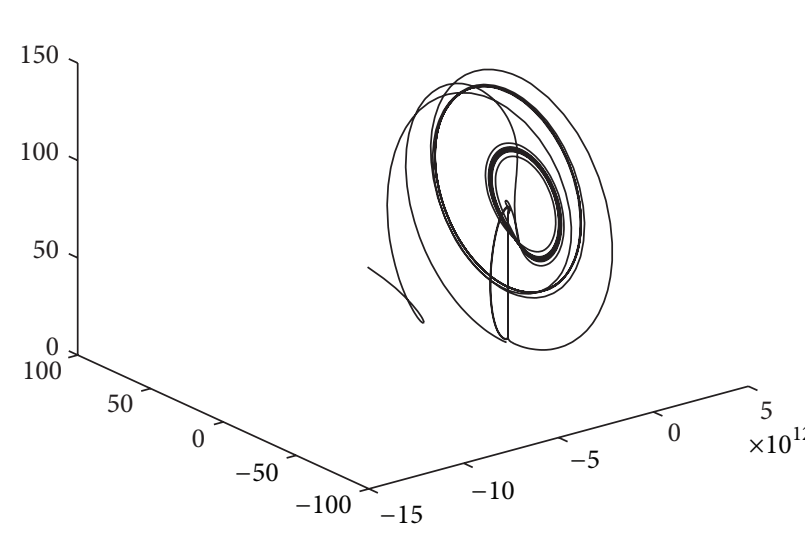

(a) System (45)-(40)

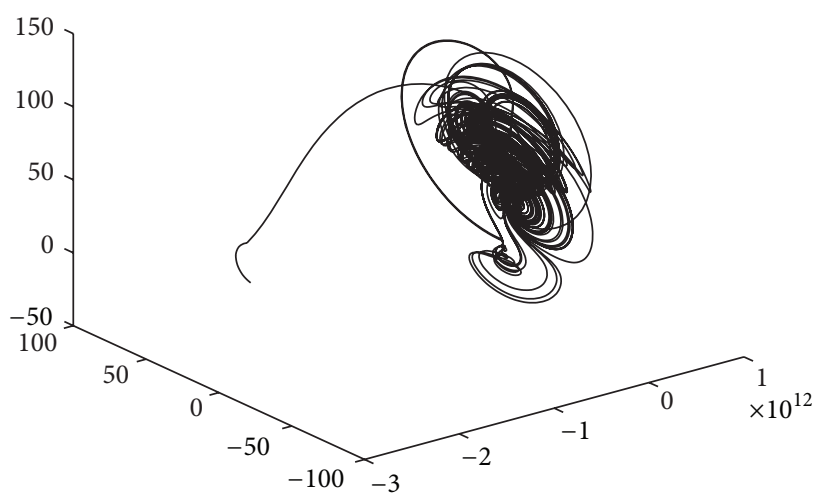

(c) System (45)-(44)

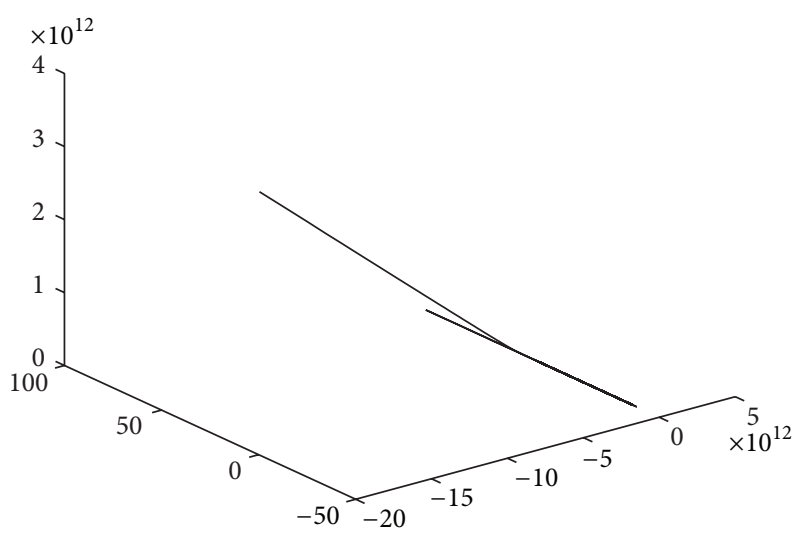

(b) System (45)-(43)

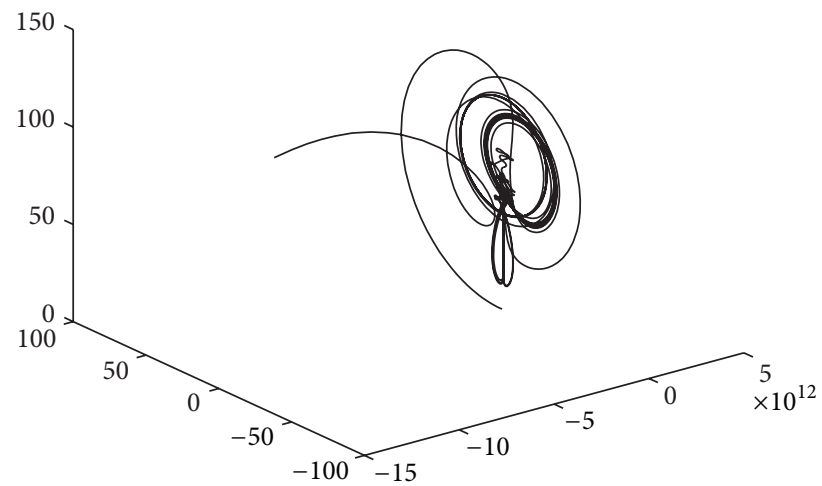

(d) System (45)-(46)

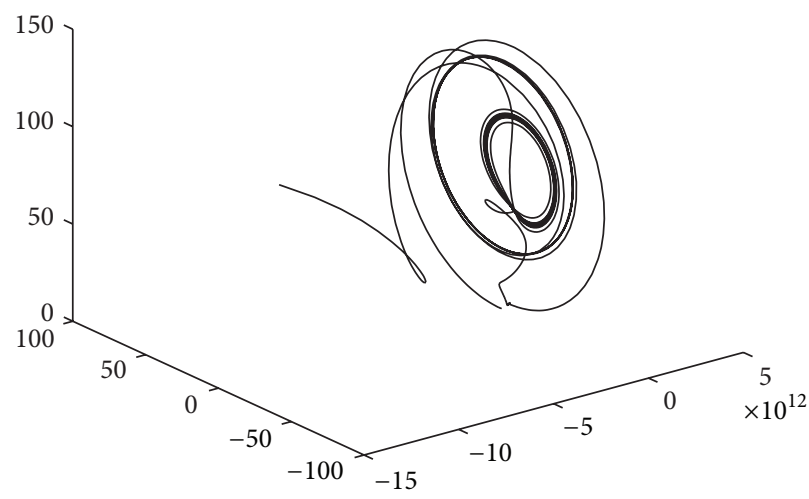

(e) System (45)-(47)

FIGURE 10: Switched system between Example (45) and others.

Example 17. The chaotic system shown in Figure 6 is

$$
\begin{aligned}
& \dot{x}=\left(\frac{20}{7}\right) x-y z+9, \\
& \dot{y}=-10 y+x z+0.5 z^{2}, \\
& \dot{z}=-4 z+x y+y z .
\end{aligned}
$$

Note. When we analyse Examples 13 to 17 by the previous means, for $\sup _{X \in R^{3}} f(\mu, X)=+\infty$, the globally exponentially attractive set and positive invariant set of them have not been obtained. The globally exponentially attractive set and positive invariant set really exist by their trajectories. Particularly, by Lü et al. chaotic system [11] and Example 17 we conjecture that they have globally conditional exponentially attractive set and positive invariant set, according to preliminary study. These are waiting for us to do further research. Meanwhile, we can compute that the maximum Lyapunov exponents of Examples 12-17 are 1.06, 0.02, 1.84, 0.01, 0.92, and 0.95, respectively. 


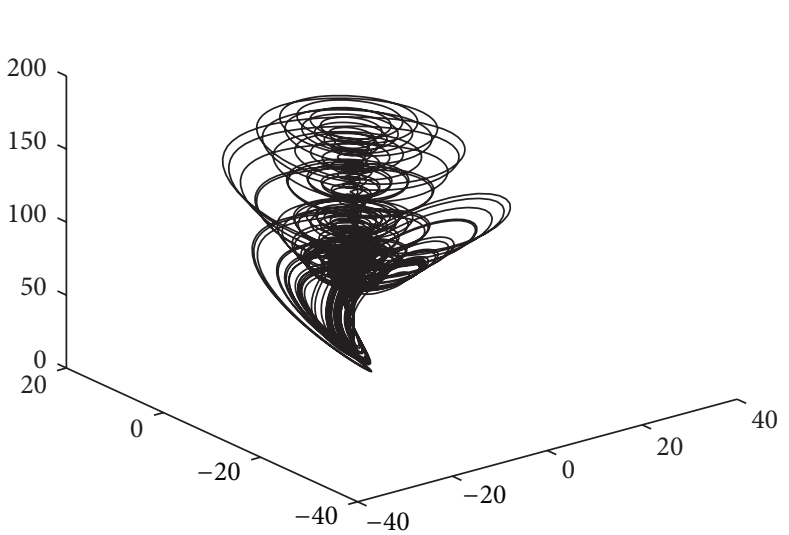

(a) System (46)-(40)

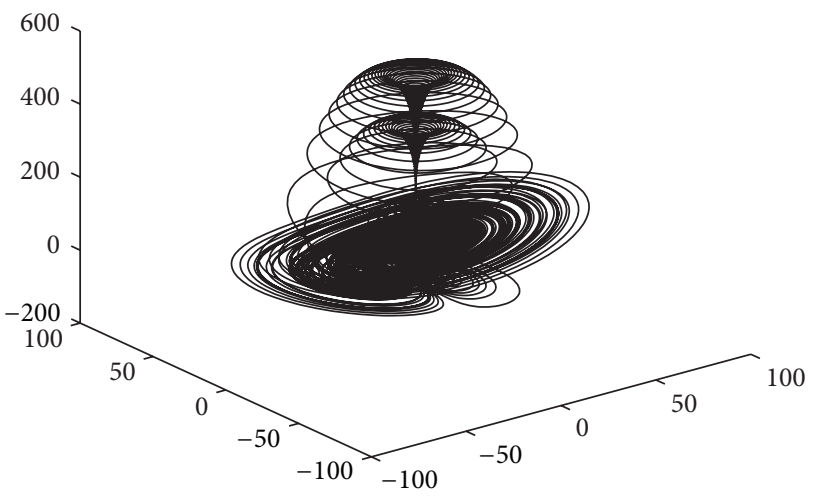

(c) System (46)-(44)

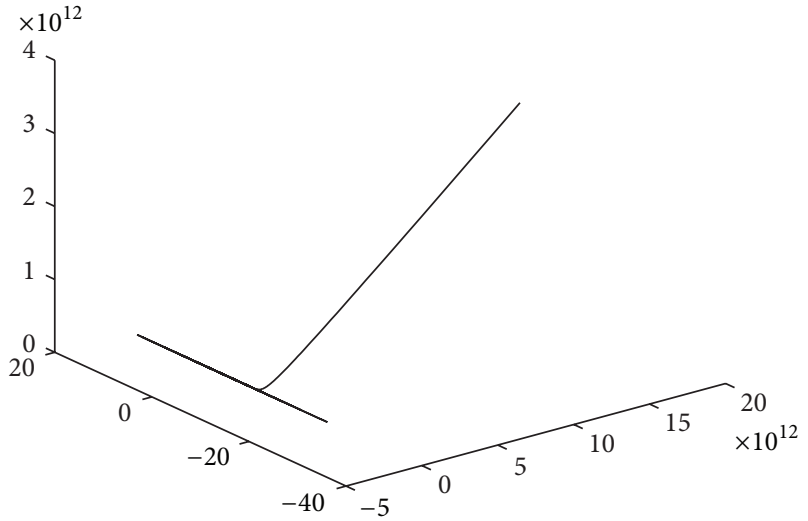

(b) System (46)-(40)

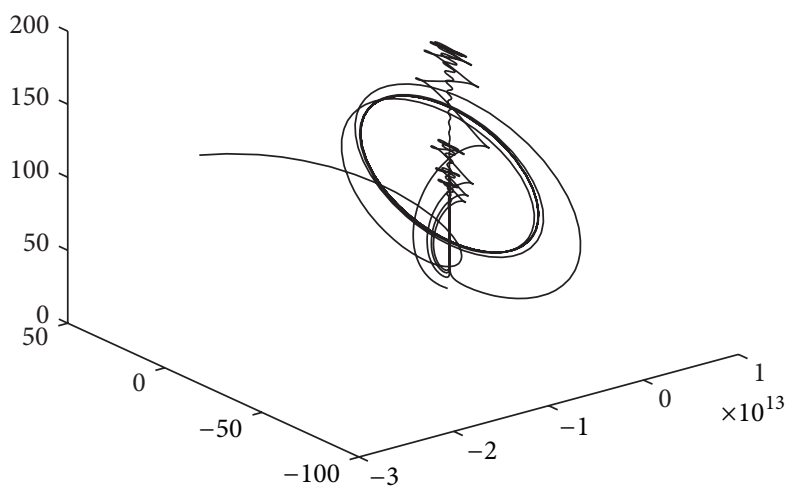

(d) System (46)-(45)

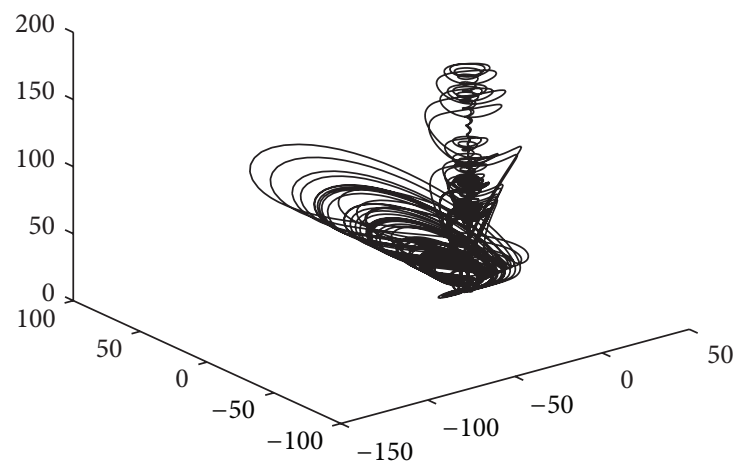

(e) System (46)-(47)

FIGURE 11: Switched system between Example (46) and others.

\section{Simulation of Switched System}

In this section, we will show some simulation results of the following switching system

$$
\dot{x}=A_{\sigma} x+f_{\sigma}(x)+C_{\sigma},
$$

where $x^{T}=\left(x_{1}, x_{2}, x_{3}\right), \sigma$ is the switching law, and

$$
\begin{gathered}
A_{\sigma}=\left[\begin{array}{ccc}
a_{11}^{\sigma} & a_{12}^{\sigma} & a_{13}^{\sigma} \\
a_{21}^{\sigma} & a_{22}^{\sigma} & a_{23}^{\sigma} \\
a_{31}^{\sigma} & a_{32}^{\sigma} & a_{33}^{\sigma}
\end{array}\right], \quad C_{\sigma}=\left[\begin{array}{c}
c_{1}^{\sigma} \\
c_{2}^{\sigma} \\
c_{3}^{\sigma}
\end{array}\right], \\
f_{\sigma}(x)=\left[\begin{array}{c}
x^{T} B_{1}^{\sigma} x \\
x^{T} B_{2}^{\sigma} x \\
x^{T} B_{3}^{\sigma} x
\end{array}\right]
\end{gathered}
$$

with $a_{i j}^{\sigma}, b_{i j k}^{\sigma}, c_{i}^{\sigma} \in R, i, j, k=1,2,3$. Each pair of $\left(A_{\sigma}, C_{\sigma}, B_{1}^{\sigma}\right.$, $\left.B_{2}^{\sigma}, B_{3}^{\sigma}\right)$ takes the form from Example 8 to Example 14. The switching law is that the system will stay in each subsystem for a constant time. In the following, we assume that (a, b) denotes a switched system which switches between system (a) and system (b). It can be seen from Figures 7 to 12 that the switched systems $(18,20),(18,22),(18,23),(20,18),(20,22)$, $(20,23),(22,18),(22,20),(22,23),(23,18),(23,20)$, and $(23,22)$ can also yield chaotic systems.

\section{Conclusion}

In this paper, the methods in [19-21] have been extended to study the globally exponentially or globally conditional 


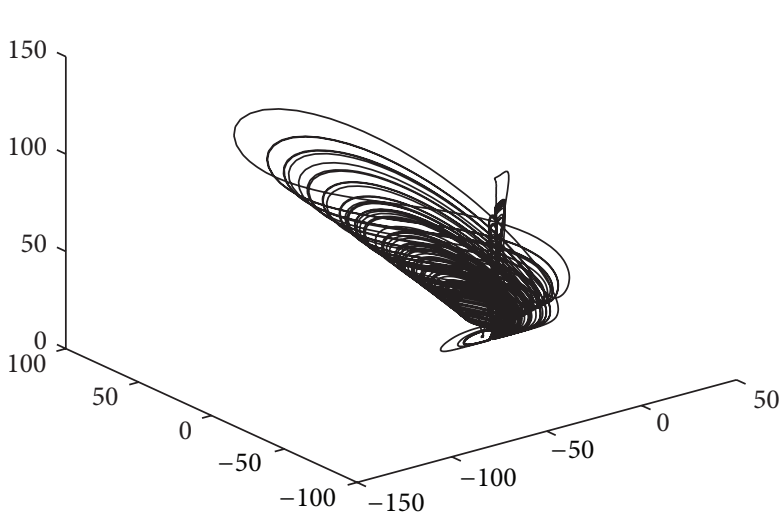

(a) System (47)-(40)

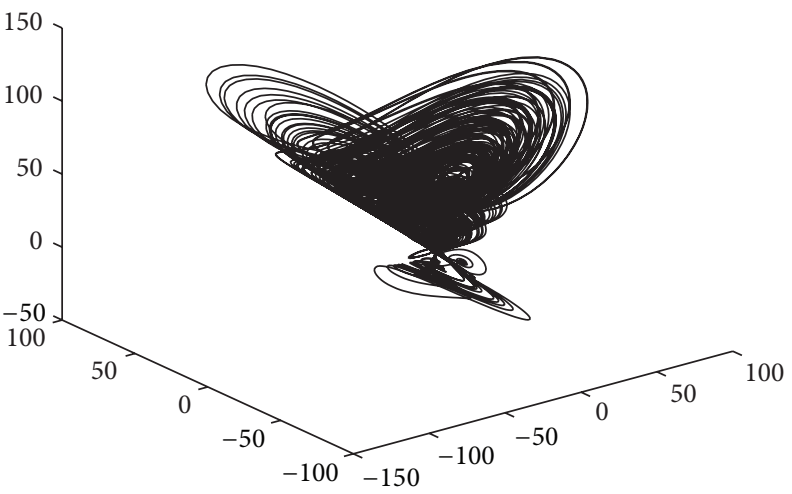

(c) System (47)-(44)

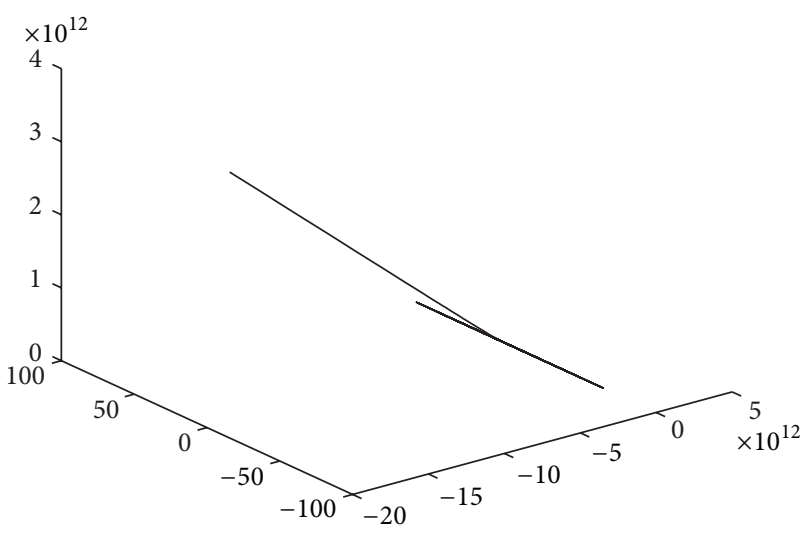

(b) System (47)-(43)

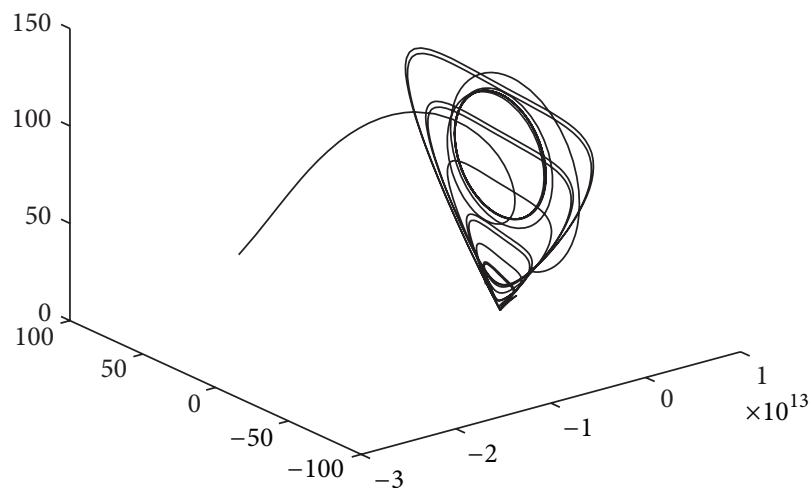

(d) System (47)-(45)

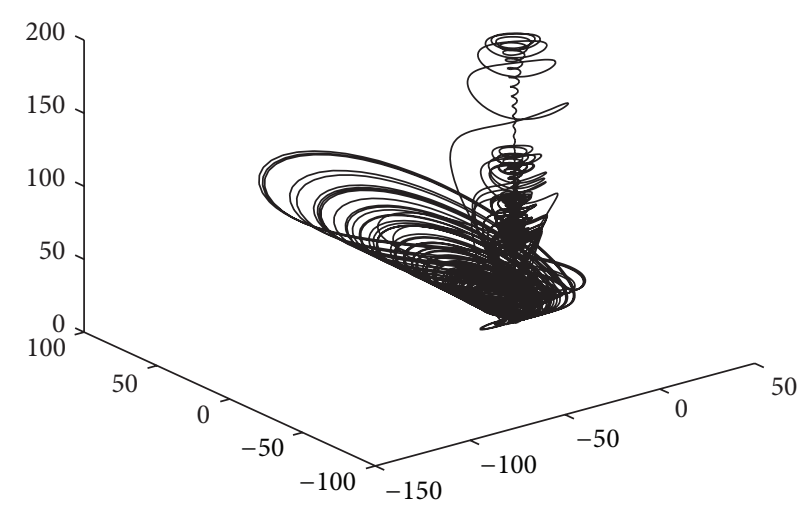

(e) System (47)-(46)

FIGURE 12: Switched system between Example (47) and others.

exponentially attractive set and positive invariant set of the three-dimensional chaotic system family with cross-product nonlinearities. We have given two theorems for studying this question and given some examples to show that such system indeed has the globally exponentially or globally conditional exponentially attractive set and positive invariant set, and the exponential estimation is explicitly derived. We have also suggested an idea to construct the chaotic systems, and some new chaotic systems have been illustrated. The simulation results are given for switched system between these new chaotic systems. It is very interesting to further research that the Hesse matrix of the $f(\mu, X)$ is not a negative definite matrix, and the dynamic system (1) is a second class threedimensional chaotic system with cross-product nonlinearities.

\section{Acknowledgments}

This work is supported by the Fundamental Research Funds for the Central Universities, China Postdoctoral Science Foundation funded project under Grant 2012M511615, National Natural Science Foundation of China under Grants 60474011 and 60904005, and the Hubei Provincial Natural Science Foundation of China under Grant 2009CDB026. 


\section{References}

[1] L. O. Chua, "Chua’s circuit: an overview ten years later," Journal of Circuits, Systems, and Computers, vol. 4, pp. 117-159, 1994.

[2] E. N. Lorenz, "Deterministic nonperiodic flow," Journal of the Atmospheric Sciences, vol. 20, pp. 130-141, 1963.

[3] T. Lofaro, "A model of the dynamics of the Newton-Leipnik attractor," International Journal of Bifurcation and Chaos, vol. 7, no. 12, pp. 2723-2733, 1997.

[4] N. A. Magnitskii and S. V. Sidorov, "A new view of the Lorenz attractor," Differential Equations, vol. 37, no. 11, pp. 1568-1579, 2001.

[5] O. E. Rőssler, "An equation for continuous chaos," Physics Letters A, vol. 15, pp. 397-398, 1976.

[6] A. M. Rucklidge, "Chaos in models of double convection," Journal of Fluid Mechanics, vol. 237, pp. 209-229, 1992.

[7] G. Chen and T. Ueta, "Yet another chaotic attractor," International Journal of Bifurcation and Chaos, vol. 9, no. 7, pp. 14651466, 1999.

[8] S. Čelikovský and G. Chen, "On a generalized Lorenz canonical form of chaotic systems," International Journal of Bifurcation and Chaos, vol. 12, no. 8, pp. 1789-1812, 2002.

[9] T. Ueta and G. Chen, "Bifurcation analysis of Chen's equation," International Journal of Bifurcation and Chaos, vol. 10, no. 8, pp. 1917-1931, 2000.

[10] W. Liu and G. Chen, "A new chaotic system and its generation," International Journal of Bifurcation and Chaos, vol. 13, no. 1, pp. 261-267, 2003.

[11] J. Lü, G. Chen, and D. Cheng, "A new chaotic system and beyond: the generalized Lorenz-like system," International Journal of Bifurcation and Chaos, vol. 14, no. 5, pp. 1507-1537, 2004.

[12] P. Wang, D. Li, X. Wu, J. Lü, and X. Yu, "Ultimate bound estimation of a class of high dimensional quadratic autonomous dynamical systems," International Journal of Bifurcation and Chaos, vol. 21, no. 9, pp. 2679-2694, 2011.

[13] P. Wang, D. Li, and Q. Hu, "Bounds of the hyper-chaotic Lorenz-Stenflo system," Communications in Nonlinear Science and Numerical Simulation, vol. 15, no. 9, pp. 2514-2520, 2010.

[14] J. M. Sarabia, E. Gómez-Déniz, M. Sarabia, and F. Prieto, "A general method for generating parametric Lorenz and Leimkuhler curves," Journal of Informetrics, vol. 4, no. 4, pp. 524-539, 2010.

[15] W. G. Hoover, "Remark on "some simple chaotic flows'”, Physical Review E, vol. 51, no. 1, pp. 759-760, 1995.

[16] R. Genesio and A. Tesi, "Harmonic balance methods for the analysis of chaotic dynamics in nonlinear systems," Automatica, vol. 28, no. 3, pp. 531-548, 1992.

[17] A. L. Shil'nikov, "On bifurcations of the Lorenz attractor in the Shimizu-Morioka model," Physica D, vol. 62, no. 1-4, pp. 338346, 1993.

[18] A. Shilnikov, G. Nicolis, and C. Nicolis, "Bifurcation and predictability analysis of a low-order atmospheric circulation model," International Journal of Bifurcation and Chaos, vol. 5, pp. 17011-17711, 1995.

[19] X. Liao, H. Luo, Y. Fu, S. Xie, and P. Yu, "On the globally exponentially attractive sets of the family of Lorenz systems," Science in China. Series E, vol. 37, pp. 715-769, 2007.

[20] X. Liao, Y. Fu, S. Xie, and P. Yu, "Globally exponentially attractive sets of the family of Lorenz systems," Science in China. Series F, vol. 51, no. 3, pp. 283-292, 2008.

[21] P. Yu, X. X. Liao, S. L. Xie, and Y. L. Fu, "A constructive proof on the existence of globally exponentially attractive set and positive invariant set of general Lorenz family," Communications in Nonlinear Science and Numerical Simulation, vol. 14, no. 7, pp. 2886-2896, 2009. 


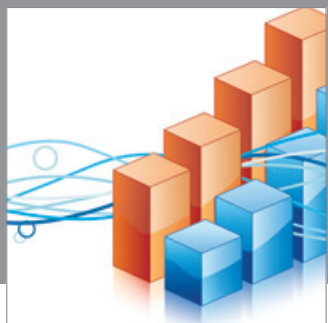

Advances in

Operations Research

mansans

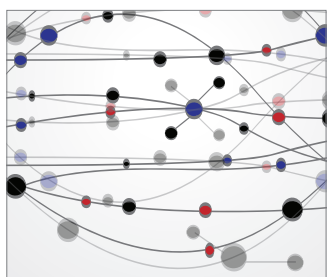

The Scientific World Journal
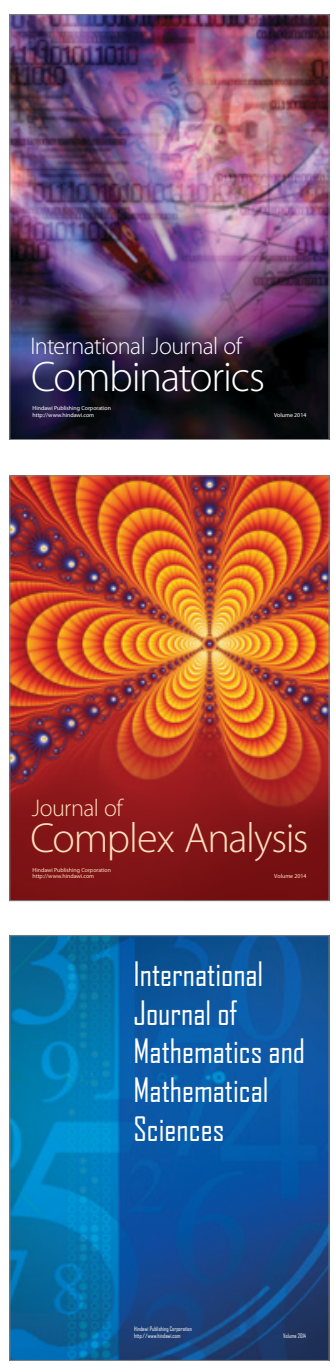
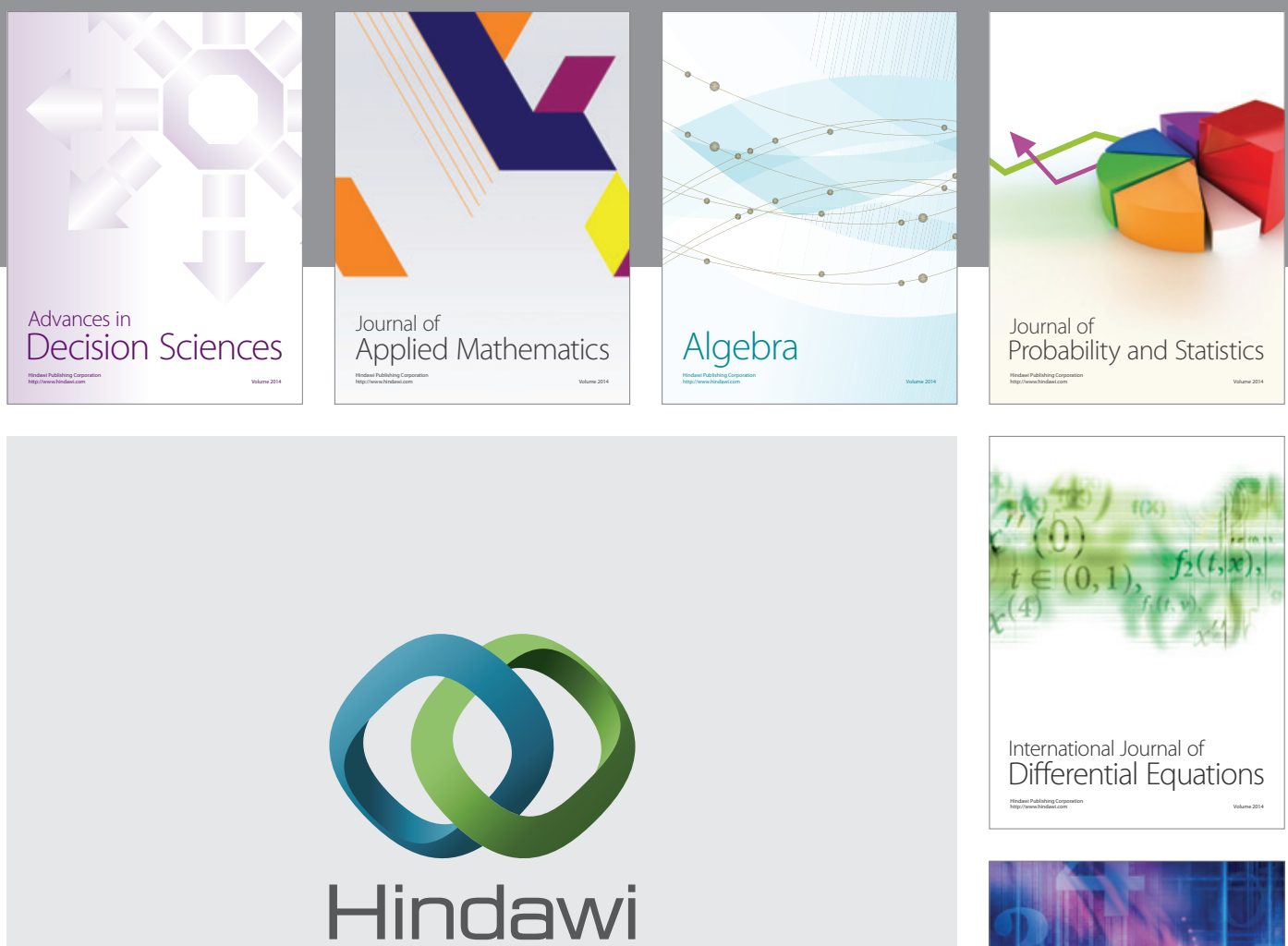

Submit your manuscripts at http://www.hindawi.com
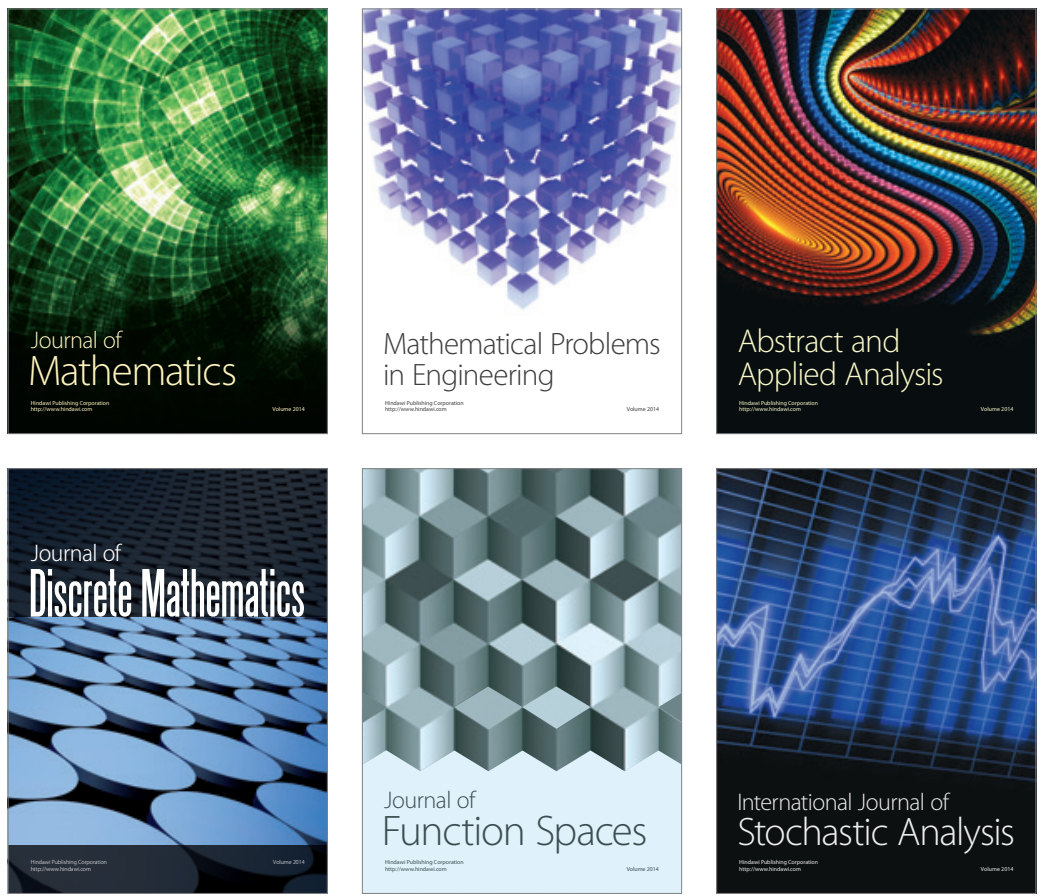

Journal of

Function Spaces

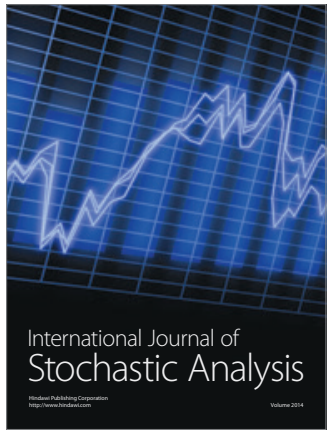

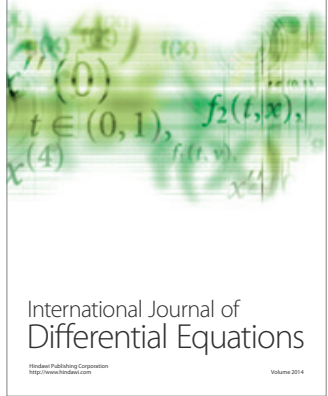
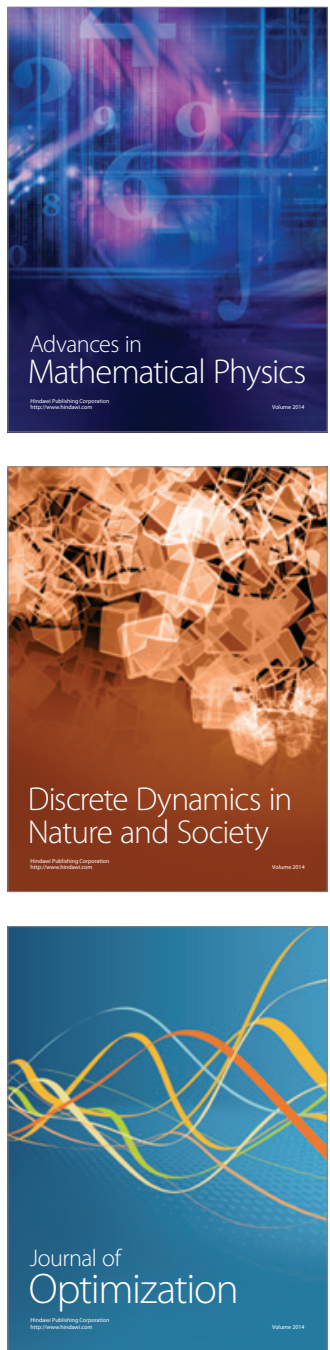